Pacific

Journal of

Mathematics

A COMBINATORIAL APPROACH TO THE QUANTIFICATION OF LIE ALGEBRAS

V.K. KhARChENKo

Volume $203 \quad$ No. 1

March 2002 


\title{
A COMBINATORIAL APPROACH TO THE QUANTIFICATION OF LIE ALGEBRAS
}

\author{
V.K. KHARChENKO
}

\begin{abstract}
We propose a notion of a quantum universal enveloping algebra for any Lie algebra defined by generators and relations which is based on the quantum Lie operation concept. This enveloping algebra has a PBW basis that admits a monomial crystallization by means of the Kashiwara idea. We describe all skew primitive elements of the quantum universal enveloping algebras for the classical nilpotent algebras of the infinite series defined by the Serre relations and prove that the above set of PBW-generators for each of these enveloping algebras coincides with the Lalonde-Ram basis of the ground Lie algebra with a skew commutator in place of the Lie operation. The similar statement is valid for Hall-Shirshov basis of any Lie algebra defined by one relation, but it is not so in the general case.
\end{abstract}

\section{Introduction.}

Quantum universal enveloping algebras appeared in the famous papers by Drinfeld [15] and Jimbo [18]. Since then a great deal of articles and number of monographs were devoted to their investigation. All of these publications are mainly concerned with a particular quantification of Lie algebras of the classical series. This is accounted for first by the fact that these Lie algebras have applications and visual interpretations in physical speculations, and then by the fact that a general, and commonly accepted as standard, notion of a quantum universal enveloping algebra is not elaborated yet (see a detailed discussion in $[\mathbf{2}],[\mathbf{3 3}])$.

In the present paper we propose a combinatorial approach to a solution of this problem by means of the quantum (Lie) operation concept [22], $[\mathbf{2 4}],[\mathbf{2 5}]$. In line with the main idea of our approach, the skew primitive elements must play the same role in quantum enveloping algebras as the primitive elements do in the classical case. By the Friedrichs criteria [13], $[\mathbf{1 6}],[32],[34],[35]$, the primitive elements form the ground Lie algebra in the classical case. For this reason we consider the space spanned by the skew primitive elements and equipped with the quantum Lie operations as a quantum analogue of a Lie algebra. 
In the second section we adduce the main notions and consider some examples. These examples, in particular, show that the Drinfeld-Jimbo enveloping algebra as well as its modifications are quantum enveloping algebras in our sense.

In the third section with the help of the Heyneman-Radford theorem we introduce a notion of a combinatorial rank of a Hopf algebra generated by skew primitive semi-invariants. Then we define the quantum enveloping algebra of an arbitrary rank that slightly generalizes the definitions given in the preceding section.

The basis construction problem for the quantum enveloping algebras is considered in the fourth section. We indicate two main methods for the construction of $P B W$-generators. One of them modifies the Hall-Shirshov basis construction process by means of replacing the Lie operation with a skew commutator. The set of the PBW-generators defined in this way, the values of hard super-letters, plays the same role as the basis of the ground Lie algebra does in the PBW theorem. At first glance it would seem reasonable to consider the $\mathbf{k}[G]$-module generated by the values of hard super-letters as a quantum Lie algebra. However, this extremely important module falls far short of being uniquely defined. It essentially depends on the ordering of the main generators, their degrees, and it is almost never antipode stable. Also we have to note the following important fact. Our definition of the hard super-letter is not constructive and, of course, it cannot be constructive in general. The basis construction problem includes the word problem for Lie algebras defined by generators and relations, while the latter one has no general algorithmic solution (see [5], [8]).

The second method is connected with the Kashiwara crystallization idea $[\mathbf{2 0}],[\mathbf{2 1}]$ (see also a development in [12], [27]). M. Kashiwara has considered the main parameter $q$ of the Drinfeld-Jimbo enveloping algebra as a temperature of some physical medium. When the temperature tend to zero, the medium crystallizes. The PBW-generators must crystallize as well. In our case under this process no one limit quantum enveloping algebra appears since the existence conditions normally include equalities of the form $\prod p_{i j}=1$ (see [24]). Nevertheless if we equate all quantification parameters to zero, the hard super-letters would form a new set of PBW-generators for the given quantum universal enveloping algebra. To put this another way, the PBW-basis defined by the super-letters admits a crystallization by means of the Kashiwara idea.

In the fifth section we bring a way to construct a Groebner-Shirshov relations system for a quantum enveloping algebra. This system is related to the main skew primitive generators, and, according to the Diamond Lemma (see $[4],[6],[41]$ ), it determines the basis appeared in the above crystallization process. The usefulness of the Groebner-Shirshov systems depends upon the fact that such a system not only defines a basis of an associative 
algebra, but it also provides a simple diminishing algorithm for expansion of elements on this basis (see, for example [3], [8]).

In the sixth section we adapt a well-known method of triangular splitting to the quantification with constants. The original method appeared in studies of simple finite dimensional Lie algebras. Then it has been extended into the field of quantum algebra in a lot of publications (see, for example [9], $[31],[42])$. By means of this method the investigation of the Drinfeld-Jimbo enveloping algebra amounts to a consideration of its positive and negative homogeneous components, quantum Borel sub-algebras. Constructions of this type also appear in classification theorems for pointed Hopf algebras (see $[\mathbf{1}])$.

In the seventh section we consider more thoroughly the quantum universal enveloping algebras of nilpotent algebras of the series $A_{n}, B_{n}, C_{n}, D_{n}$ defined by the Serre relations. We adduce first lists of all hard super-letters in the explicit form, then Groebner-Shirshov relations systems, and next spaces $L\left(U_{P}(\mathfrak{g})\right)$ spanned by the skew primitive elements (i.e., the Lie algebra quantifications $\mathfrak{g}_{P}$ proper). In all cases the lists of hard super-letters (but not the hard super-letters themselves) turn out to be independent of the quantification parameters. This means that the PBW-generators result from the Hall-Shirshov basis of the ground Lie algebra by replacing the Lie operation with the skew commutator. The same is valid for the GroebnerShirshov relations systems. Note that the Hall-Shirshov bases, under the name standard Lyndon bases, for the classical Lie series were constructed by P. Lalonde and A. Ram [28], while the Groebner-Shirshov systems of Lie relations were found by L.A. Bokut' and A.A. Klein [7].

Furthermore, in all cases $\mathfrak{g}_{P}$ as a quantum Lie algebra (in our sense) proves to be very simple in structure. Either it is a colored Lie super-algebra (provided that the parameter $p_{11}$ equals 1 ), or values of all non-unary quantum Lie operations equal zero on $\mathfrak{g}_{P}$. In particular, if $\operatorname{char}(\mathbf{k})=0$ and $p_{11}^{t} \neq 1$ then the quantum Lie operations may be defined on $\mathfrak{g}_{P}$, but all of them have zero values. Thus, in this case we have a reason to consider $U_{P}(\mathfrak{g})$ as an algebra of 'commutative' quantum polynomials, since the universal enveloping algebra of a Lie algebra with zero bracket is the algebra of ordinary commutative polynomials. Immediately afterwards a number of interesting questions appears. What is the structure of other algebras of 'commutative' quantum polynomials? When do the PBW-generators result from a basis of the ground Lie algebra by means of replacing the Lie operation with the skew commutator? These and other questions we briefly discuss in the last section.

It is well to bear in mind that the combinatorial approach is not free from flaws: The quantum universal enveloping algebra essentially depends on a combinatorial representation of the ground Lie algebra, i.e., a close connection with the abstract category of Lie algebras is lost. 


\section{Quantum enveloping algebras.}

Recall that a variable $x$ is called a quantum variable if an element $g_{x}$ of a fixed Abelian group $G$ and a character $\chi^{x} \in G^{*}$ are associated with it. The parameters $g_{x}$ and $\chi^{x}$ associated with a quantum variable say that an element $a$ in a Hopf algebra $H$ may be considered as a value of this quantum variable only if $a$ is a skew primitive semi-invariant with the same parameters, that is

$$
\Delta(a)=a \otimes 1+g_{x} \otimes a, \quad g^{-1} a g=\chi^{x}(g) a, \quad g \in G,
$$

where we suppose that the elements of $G$ have some interpretation in $H$ as grouplike elements.

A noncommutative polynomial in quantum variables is called a quantum Lie operation if all of its values in all Hopf algebras are skew primitive for all values of the quantum variables.

Let $x_{1}, \ldots, x_{n}$ be a set of quantum variables. For each word $u$ in $x_{1}, \ldots, x_{n}$ we denote by $g_{u}$ an element of $G$ that appears from $u$ by replacing of all $x_{i}$ with $g_{x_{i}}$. In the same way we denote by $\chi^{u}$ a character that appears from $u$ by replacing of all $x_{i}$ with $\chi^{x_{i}}$. Thus on the free algebra $\mathbf{k}\left\langle x_{1}, \ldots, x_{n}\right\rangle$ a grading by the group $G \times G^{*}$ is defined. For each pair of homogeneous elements $u, v$ we fix the denotations $p_{u v}=\chi^{u}\left(g_{v}\right)=p(u, v)$.

We define an action of $G$ on $\mathbf{k}\left\langle x_{1}, \ldots, x_{n}\right\rangle$ by $g^{-1} u g=\chi^{u}(g) u$, where $u$ is an arbitrary monomial in $x_{1}, \ldots x_{n}$. The skew group algebra $G\langle X\rangle=$ $\mathbf{k}\left\langle x_{1}, \ldots, x_{n}\right\rangle * G$ has a natural Hopf algebra structure with the coproduct

$$
\Delta\left(x_{i}\right)=x_{i} \otimes 1+g_{x_{i}} \otimes x_{i}, \quad 1 \leq i \leq 1, \quad \Delta(g)=g \otimes g, g \in G .
$$

Hence $x_{i}=x_{i} \in G\langle X\rangle$ are correct values of quantum variables. By this means the quantum Lie operations can be identified with skew primitive polynomials in $G\langle X\rangle$. Recall that the Hopf algebra $G\langle X\rangle$ is called the free enveloping algebra for the set $X$ of quantum variables (see [22, Sect. 3] under denotation $H\langle X\rangle)$.

The free algebra $\mathbf{k}\left\langle x_{1}, \ldots, x_{n}\right\rangle$ has a structure of braided bigraded Hopf algebra. Namely, let $\mathcal{H}$ be an associative algebra graded by the group $G \times G^{*}$ :

$$
\mathcal{H}=\sum_{g \in G, \chi \in G^{*}} \oplus \mathcal{H}_{g}^{\chi}
$$

Define multiplication on the tensor product $\mathcal{H} \otimes \mathcal{H}$ of linear spaces by setting

$$
(a \otimes b) \cdot(c \otimes d)=\left(\chi^{c}\left(g_{b}\right)\right)^{-1}(a c \otimes b d) .
$$

The result is an associative algebra, denoted by $\mathcal{H} \underline{\otimes} \mathcal{H}$. Now if, in the definition of a Hopf algebra, we change the sign $\otimes$ by $\underline{\otimes}$, and assume coproduct, $\Delta^{b}$, counity, $\varepsilon^{b}$, and antipode, $S^{b}$, are homogeneous, we arrive at a definition of the braided bigraded Hopf algebra. In other words a braided 
bigraded Hopf algebra is a graded by $G \times G^{*}$ Hopf algebra in braided category where the braiding is connected with the grading by the formula $c(u \otimes v)=\left(\chi^{v}\left(g_{u}\right)\right)^{-1}(v \otimes u)$.

The quantum Lie operation can be defined equivalently as a $G \times 1$ homogeneous polynomial that has only primitive values in all braided bigraded Hopf algebras provided that the correct value of a quantum variable $x=x_{g}^{\chi}$ is primitive and homogeneous, that is $a \in \mathcal{H}_{g}^{\chi}, \Delta^{b}(a)=a \underline{\otimes} 1+1 \underline{\otimes} a$. The detailed discussion of the notion of quantum Lie operation and examples can be found in [22, Sect. 1-4].

Recall that a constitution of a word $u$ is a sequence of nonnegative integers $\left(m_{1}, m_{2}, \ldots, m_{n}\right)$ such that $u$ is of degree $m_{1}$ in $x_{1}, \operatorname{deg}_{1}(u)=m_{1}$; of degree $m_{2}$ in $x_{2}, \operatorname{deg}_{2}(u)=m_{2}$; and so on (see [39, Definition 3]). Since the group $G$ is Abelian, all constitution homogeneous polynomials are homogeneous with respect to the grading. Let us define a bilinear skew commutator on the set of graded homogeneous noncommutative polynomials by the formula

$$
[u, v]=u v-p_{u v} v u .
$$

These brackets satisfy the following Jacobi and skew differential identities:

$$
\begin{gathered}
{[[u, v], w]=[u,[v, w]]+p_{w v}^{-1}[[u, w], v]+\left(p_{v w}-p_{w v}^{-1}\right)[u, w] \cdot v ;} \\
{[[u, v], w]=[u,[v, w]]+p_{v w}[[u, w], v]+p_{u v}\left(p_{v w} p_{w v}-1\right) v \cdot[u, w] ;}
\end{gathered}
$$

$$
[u, v \cdot w]=[u, v] \cdot w+p_{u v} v \cdot[u, w] ; \quad[u \cdot v, w]=p_{v w}[u, w] \cdot v+u \cdot[v, w],
$$

where by the dot we denote the usual multiplication. It is easy to see that the following conditional restricted identities are valid as well

$$
\left[u, v^{n}\right]=[\ldots[[u, v], v] \ldots, v] ; \quad\left[v^{n}, u\right]=[v,[\ldots[v, u] \ldots]],
$$

provided that $p_{v v}$ is a primitive $t$-th root of unit, and $n=t$ or $n=t l^{k}$ in the case of characteristic $l>0$.

Suppose that a Lie algebra $\mathfrak{g}$ is defined by the generators $x_{1}, \ldots, x_{n}$ and the relations $f_{i}=0$. Let us convert the generators into quantum variables. For this associate to them elements of $G \times G^{*}$ in arbitrary way. Let $P=$ $\left\|p_{i j}\right\|, p_{i j}=\chi^{x_{i}}\left(g_{x_{j}}\right)$ be the quantification matrix.

Definition 2.1. A braided quantum enveloping algebra of $\mathfrak{g}$ is a braided bigraded Hopf algebra $U_{P}^{b}(\mathfrak{g})$ defined by the variables $x_{1}, \ldots, x_{n}$ and the relations $f_{i}=0$, where the Lie operation is replaced with (2), provided that in this way $f_{i}$ are converted into the quantum Lie operations $f_{i}^{*}$. The coproduct and the braiding are defined by

$$
\begin{aligned}
\Delta^{b}\left(x_{i}\right) & =x_{i} \underline{\otimes} 1+1 \underline{\otimes} x_{i}, \\
\left(x_{i} \underline{\otimes} x_{j}\right) \cdot\left(x_{k} \underline{\otimes} x_{m}\right) & =\left(\chi^{x_{k}}\left(g_{x_{j}}\right)\right)^{-1} x_{i} x_{k} \underline{\otimes} x_{j} x_{m} .
\end{aligned}
$$


Definition 2.2. A simple quantification of $U(\mathfrak{g})$ or a quantum universal enveloping algebra of $\mathfrak{g}$ is an algebra $U_{P}(\mathfrak{g})$ that is isomorphic to the skew group algebra

$$
U_{P}(\mathfrak{g})=U_{P}^{b}(\mathfrak{g}) * G,
$$

where the group action and the coproduct are defined by

$$
g^{-1} x_{i} g=\chi^{x_{i}}(g) x_{i}, \Delta\left(x_{i}\right)=x_{i} \otimes 1+g_{x_{i}} \otimes x_{i}, \Delta(g)=g \otimes g .
$$

Definition 2.3. A quantification with constants is a simple quantification where additionally some generators $x_{i}$ associated to the trivial character are replaced with the constants $\alpha_{i}\left(1-g_{x_{i}}\right)$.

The formulae (10) and (7) correctly define the coproduct since by definition of the quantum Lie operation $\Delta\left(f_{i}^{*}\right)=f_{i}^{*} \otimes 1+g_{i} \otimes f_{i}^{*}$ in the case of ordinary Hopf algebras and $\Delta^{b}\left(f_{i}^{*}\right)=f_{i}^{*} \otimes 1+1 \otimes f_{i}^{*}$ in the braided case.

We have to note that the defined quantifications essentially depend on the combinatorial representation of the Lie algebra. For example, an additional relation $\left[x_{1}, x_{1}\right]=0$ does not change the Lie algebra. At the same time if $\chi^{x_{1}}\left(g_{1}\right)=-1$ then this relation admits the quantification and yields a nontrivial relation for the quantum enveloping algebra, $2 x_{1}^{2}=0$.

Example 1. Suppose that the Lie algebra is defined by a system of constitution homogeneous relations. If the characters $\chi^{i}$ are such that $p_{i j} p_{j i}=1$ for all $i, j$ then the skew commutator itself is a quantum operation. Therefore on replacing the Lie operation all relations become quantum operations as well. This means that the braided enveloping algebra is the universal enveloping algebra $U\left(\mathfrak{g}^{\mathrm{col}}\right)$ of the colored Lie super-algebra which is defined by the same relations as the given Lie algebra is. The simple quantification appears as the Radford biproduct $U\left(\mathfrak{g}^{\mathrm{col}}\right) \star \mathbf{k}[G]$ or, equivalently, as the universal $G$-enveloping algebra of the colored Lie super-algebra $\mathfrak{g}^{\text {col }}$ (see [37] or [22, Example 1.9]).

Example 2. Suppose that the Lie algebra $\mathfrak{g}$ is defined by the generators $x_{1}, \ldots, x_{n}$ and the system of nil relations

$$
x_{j}\left(a d x_{i}\right)^{n_{i j}}=0, \quad 1 \leq i \neq j \leq n .
$$

Usually instead of the matrix of degrees (without the main diagonal), $\left\|n_{i j}\right\|$, the matrix $A=\left\|a_{i j}\right\|, a_{i j}=1-n_{i j}$ is considered. The Coxeter graph $\Gamma(A)$ is associated to every such a matrix. This graph has the vertices $1, \ldots, n$, where the vertex $i$ is connected by $a_{i j} a_{j i}$ edges with the vertex $j$.

If $a_{i j}=0$ then the relation $x_{j} \mathrm{ad} x_{i}=0$ is in the list (11), and the relation $x_{i}\left(\operatorname{ad} x_{j}\right)^{n_{j i}}=0$ is a consequence of it. The skew commutator $\left[x_{j}, x_{i}\right]$ is a quantum Lie operation if and only if $p_{i j} p_{j i}=1$. Under this condition we have $\left[x_{i}, x_{j}\right]=-p_{i j}\left[x_{j}, x_{i}\right]$. Therefore both in the given Lie algebra and in its quantification one may replace the relation $x_{i}\left(\operatorname{ad} x_{j}\right)^{n_{j i}}=0$ with $x_{i} \operatorname{ad} x_{j}=0$. 
In other words, without loss of generality, we may suppose that $a_{i j}=0 \leftrightarrow$ $a_{j i}=0$. By the Gabber-Kac theorem [17] we get that the algebra $\mathfrak{g}$ is the positive homogeneous component $\mathfrak{g}_{1}^{+}$of a Kac-Moody algebra $\mathfrak{g}_{1}$.

The following theorem describes the conditions for a homogeneous polynomial in two variables which is linear in one of them to be a quantum operation.

Theorem 2.4. For quantum variables $x_{1}$ and $x_{2}$, there exists a nonzero linear in $x_{1}$ quantum Lie operation $W$ of degree $n$ in $x_{2}$ if and only if either $p_{12} p_{21}=p_{22}^{1-n}$, or $p_{22}$ is a primitive $m$-th root of unity, $m \mid n$, and $p_{12}^{m} p_{21}^{m}=1$. If one of these conditions is satisfied, then all the operations have the form $W=\alpha\left[\ldots\left[\left[x_{1} x_{2}\right] x_{2}\right] \ldots x_{2}\right], \alpha \in \mathbf{k}$, where the brackets are defined by $(2)$.

Proof. It follows from Theorem 6.1 [22], and the conditional identity (6).

From this theorem we have the following corollary.

Corollary 2.5. If $n_{i j}$ is a simple number or unit and in the former case $p_{i i}$ is not a primitive $n_{i j}$-th root of unit, then the relation (11) admits a quantification if and only if $p_{i j} p_{j i}=p_{i i}^{a_{i j}}$.

Theorem 2.4 provides no essential restrictions on the non-diagonal parameters $p_{i j}$ : If the matrix $P$ correctly defines a quantification of (11) then for every set $Z=\left\{z_{i j} \mid z_{i j} z_{j i}=z_{i i}=1\right\}$ the following matrix does as well:

$$
P_{Z}=\left\{p_{i j} z_{i j} \mid p_{i j} \in P, z_{i j} \in Z\right\} .
$$

Example 3. Let $G$ be freely generated by $g_{1}, \ldots g_{n}$ and $A$ be a generalized Cartan matrix symmetrized by $d_{1}, \ldots, d_{n}$, while the characters are defined by $p_{i j}=q^{-d_{i} a_{i j}}$. In this case the simple quantification of $\mathfrak{g}$ defined by (11) is the positive component of the Drinfeld-Jimbo enveloping algebra together with the group-like elements, $U_{P}(\mathfrak{g})=U_{q}^{+}(\mathfrak{g}) * G$. By means of an arbitrary deformation (12) one may define a 'coloring' of $U_{q}^{+}(\mathfrak{g}) * G$.

The braided enveloping algebra equals $U_{q}^{+}(\mathfrak{g})$ where the coproduct and braiding are defined by (7) and (8) with the coefficient $q^{d_{k} a_{k j}}$. The formula (12) correctly defines its 'coloring' as well.

Example 4. If in the above example we complete the set of quantum variables by the new ones $x_{1}^{-}, \ldots, x_{n}^{-} ; z_{1}, \ldots, z_{n}$ such that

$$
\chi^{x^{-}}=\left(\chi^{x}\right)^{-1}, \quad g_{x^{-}}=g_{x}, \quad \chi^{z_{i}}=\mathrm{id}, \quad g_{z_{i}}=g_{i}^{2},
$$

then, by Theorem 2.4, the Gabber-Kac relations (2), (3) of [17, Theorem 2], and $\left[e_{i}, f_{j}\right]=\delta_{i j} h_{i}$ under the identification $e_{i}=x_{i}, f_{i}=x_{i}^{-}, h_{i}=z_{i}$ admit the quantification with constants $z_{i}=\varepsilon_{i}\left(1-g_{i}^{2}\right)$. (Informally we may consider the obtained quantification as one of the Kac-Moody algebra identifying $g_{i}$ with $q^{h_{i}}$, where the rest of the Kac-Moody algebra relations, $\left[h_{i}, e_{j}\right]=a_{i j} e_{i},\left[h_{i}, f_{j}\right]=-a_{i j} f_{j}$, is quantified to the $G$-action: 
$g_{j}^{-1} x_{i}^{ \pm} g_{j}=q^{\mp d_{i j} a_{i j}} x_{i}^{ \pm}$.) This quantification coincides with the DrinfeldJimbo one under a suitable choice of $x_{i}, x_{i}^{-}$, and $\varepsilon_{i}$ depending up the particular definition of $U_{q}(\mathfrak{g})$ :

$[20] \Delta_{+} \quad x_{i}=e_{i}, g_{i}=t_{i}, x_{i}^{-}=t_{i} f_{i}, p_{i j}=q_{j}^{-\left\langle h_{j}, \alpha_{i}\right\rangle}, \varepsilon_{i}=\left(q_{i}-q_{i}^{3}\right)^{-1}$;

$[20] \Delta_{-} \quad x_{i}=f_{i}, g_{i}=t_{i}, x_{i}^{-}=e_{i} t_{i}, p_{i j}=q_{j}^{\left\langle h_{j}, \alpha_{i}\right\rangle}, \varepsilon_{i}=\left(q_{i}^{-1}-q_{i}\right)^{-1}$; [36] $\quad x_{i}=E_{i} K_{i}, g_{i}=K_{i}^{2}, x_{i}^{-}=F_{i} K_{i}, p_{i j}=q^{-2 d_{i} a_{i j}}, \varepsilon_{i}=\left(1-q^{4 d_{i}}\right)^{-1}$.

By (13) the brackets $\left[x_{i}, x_{j}^{-}\right]$are the quantum Lie operation only if $p_{i j}=p_{j i}$. So in this case the 'colorings' (12) may be only black-white, $z_{i j}= \pm 1$.

In the perfect analogy the Kang quantification [19] of the generalized Kac-Moody algebras [10] is a quantification in our sense as well.

\section{Combinatorial rank.}

Recall that a Hopf algebra $H$ is called character if the group $G$ of all grouplike elements is commutative and $H$ is generated by skew primitive semiinvariants $a_{i}$ :

$$
\Delta\left(a_{i}\right)=a_{i} \otimes 1+g_{a_{i}} \otimes a_{i}, \quad g^{-1} a_{i} g=\chi^{a_{i}}(g) a_{i}, \quad g \in G .
$$

By the definitions of the above section the quantum enveloping algebras (with or without constants) are character Hopf algebras. In this section by means of a combinatorial rank notion we identify the quantum enveloping algebras in the class of character Hopf algebras.

Let $H$ be a character Hopf algebra generated by the skew primitive semiinvariants $a_{1}, \ldots, a_{n}$. Let us associate a quantum variable $x_{i}$ with the parameters $\left(\chi^{a_{i}}, g_{a_{i}}\right)$ to $a_{i}$. Denote by $G\langle X\rangle$ the free enveloping algebra defined by the quantum variables $x_{1}, \ldots, x_{n}$. The map $x_{i} \rightarrow a_{i}$ has an extension to a homomorphism of Hopf algebras $\varphi: G\langle X\rangle \rightarrow H$. Denote by $I$ the kernel of this homomorphism. If $I \neq 0$ then by the Heyneman-Radford theorem (see [36, Corollary 5.4.7]), the Hopf ideal $I$ has a nonzero skew primitive element. Let $I_{1}$ be an ideal generated by all skew primitive elements of $I$. Clearly $I_{1}$ is a Hopf ideal as well. Now consider the Hopf ideal $I / I_{1}$ of the quotient Hopf algebra $G\langle X\rangle / I_{1}$. This ideal also has nonzero skew primitive elements (provided $I_{1} \neq I$ ). Denote by $I_{2} / I_{1}$ the ideal generated by all skew primitive elements of $I / I_{1}$, where $I_{2}$ is its preimage with respect to the projection $G\langle X\rangle \rightarrow G\langle X\rangle / I_{1}$. Continuing the process we will find a strictly increasing, finite or infinite, chain of Hopf ideals of $G\langle X\rangle$ :

$$
0=I_{0} \subset I_{1} \subset I_{2} \subset \ldots \subset I_{n} \subset \ldots, \bigcup_{\alpha} I_{\alpha}=I .
$$

Definition 3.1. The length of (15) is called a combinatorial rank of $H$. 
By definition, the combinatorial rank of any quantum enveloping algebra (with constants) equals one. In the case of zero characteristic the inverse statement is valid as well.

Theorem 3.2. Each character Hopf algebra of the combinatorial rank 1 over a field of zero characteristic is isomorphic to a quantum enveloping algebra with constants of a Lie algebra.

Proof. By definition, $I$ is generated by skew primitive elements. These elements as noncommutative polynomials are the quantum Lie operations. Consider one of them, say $f$. Let us decompose $f$ into a sum of homogeneous components $f=\sum f_{i}$. All positive components belongs to $\mathbf{k}\langle X\rangle$ and they are the quantum Lie operations themselves, while the constant component has the form $\alpha(1-g), g \in G$ (see [22, Sec. 3 and Prop. 3.3]). If $\alpha \neq 0$ then we introduce a new quantum variable $z_{f}$ with the parameters $(i d, g)$. Each $f_{i}$ has a representation through the skew commutator. Indeed, by $[\mathbf{2 2}$, Theorem 7.5$]$ the complete linearization $f_{i}^{\text {lin }}$ of $f_{i}$ has the required representation. By the identification of variables in a suitable way in $f_{i}^{\text {lin }}$ we get the required representation for $f_{i}$ multiplied by a natural number, $m_{i} f_{i}=f_{i}^{[]}$.

Now consider a Lie algebra $\mathfrak{g}$ defined by the generators $x_{i}, z_{f}$ and the relations $\sum m_{i}^{-1} f_{i}^{[]}+z_{f}=0$, with the Lie multiplication in place of the skew commutator. It is clear that $H$ is the quantification with constants of g.

In the same way one may introduce the notion of the combinatorial rank for the braided bigraded Hopf algebras. In this case all braided quantum enveloping algebras are of rank 1, and all braided bigraded algebras of rank 1 are the braided quantification of some Lie algebras.

Now we are ready to define a quantification of arbitrary rank. For this in the definitions of the above section it is necessary to change the requirement that all $f_{i}^{*}$ are quantum Lie operations with the following condition.

The set $F$ splits in a union $F=\bigcup_{j=1}^{n} F_{j}$ such that $F_{1}^{*}$ consists of quantum Lie operations; the set $F_{2}^{*}$ consists of skew primitive elements of $G\left\langle X|| F_{1}^{*}\right\rangle$; the set $F_{3}^{*}$ consists of skew primitive elements of $G\left\langle X|| F_{1}^{*}, F_{2}^{*}\right\rangle$, and so on.

The quantum enveloping algebras of an arbitrary rank are character Hopf algebras also. Conversely, if a character Hopf algebra $H$ is homogeneous and the ground field has a zero characteristic, then $H$ is a quantification of some rank of a suitable Lie algebra (see [26]). It is not clear if there exist character Hopf algebras, or braided bigraded Hopf algebras, of infinite combinatorial rank; while it is easy to see that $\bigcup_{n=1}^{\infty} I_{n}=I$. Also it is possible to show that $F_{1}$ always contains all relations of a minimal constitution in $F$. For example, each of (11) is of a minimal constitution in (11). Therefore the quantification of arbitrary rank with the identification $g_{i}=\exp \left(h_{i}\right)$ of any 
(generalized) Kac-Moody algebra $\mathfrak{g}$, or its nilpotent component $\mathfrak{g}^{+}$, is always a quantification in the sense of the above section.

\section{PBW-generators and monomial crystallization.}

The next result yields a PBW basis for the quantum enveloping algebras.

Theorem 4.1. Every character Hopf algebra $H$ has a linearly ordered set of constitution homogeneous elements $U=\left\{u_{i} \mid i \in I\right\}$ such that the set of all products $g u_{1}^{n_{1}} u_{2}^{n_{2}} \cdots u_{m}^{n_{m}}$, where $g \in G, u_{1}<u_{2}<\ldots<u_{m}, 0 \leq n_{i}<h(i)$ forms a basis of $H$. Here if $p_{i i} \stackrel{d f}{=} p_{u_{i} u_{i}}$ is not a root of unity then $h(i)=\infty$; if $p_{i i}=1$ then either $h(i)=\infty$ or $h(i)=l$ is the characteristic of the ground field; if $p_{i i}$ is a primitive $t$-th root of unity, $t \neq 1$, then $h(i)=t$.

The set $U$ is referred to as a set of $P B W$-generators of $H$. This theorem easily follows from [23, Theorem 2]. Let us recall necessary notions.

Let $a_{1}, \ldots, a_{n}$ be a set of skew primitive generators of $H$, and let $x_{i}$ be the associated quantum variables. Consider the lexicographical ordering of all words in $x_{1}>x_{2}>\ldots>x_{n}$. A beginning of a word is considered to be greater than the word itself, for example $x_{1}>x_{1} x_{2}^{2}>x_{1} x_{2}^{2} x_{1}$. A nonempty word $u$ is called standard if $v w>w v$ for each decomposition $u=v w$ with nonempty $v, w$. The following properties are well-known (see, for example $[11],[14],[29],[40],[41])$.

1s. A word $u$ is standard if and only if it is greater than each of its ends.

2s. Every standard word starts with a maximal letter that it has.

3s. Each word $c$ has a unique representation $c=u_{1}^{n_{1}} u_{2}^{n_{2}} \cdots u_{k}^{n_{k}}$, where $u_{1}<u_{2}<\cdots<u_{k}$ are standard words (the Lyndon theorem).

$4 \mathrm{~s}$. If $u, v$ are different standard words and $u^{n}$ contains $v^{k}$ as a sub-word, $u^{n}=c v^{k} d$, then $u$ itself contains $v^{k}$ as a sub-word, $u=b v^{k} e$.

Recall that a nonassociative word is a word where brackets [,] somehow arranged to show how multiplication applies. If $[u]$ denotes a nonassociative word then by $u$ we denote an associative word obtained from $[u]$ by removing the brackets (of course $[u]$ is not uniquely defined by $u$ in general).

The set of standard nonassociative words is defined as the smallest set $S L$ that contains all variables $x_{i}$ and satisfies the following properties.

1) If $[u]=[[v][w]] \in S L$ then $[v],[w] \in S L$, and $v>w$ are standard.

2) If $[u]=\left[\left[\left[v_{1}\right]\left[v_{2}\right]\right][w]\right] \in S L$ then $v_{2} \leq w$.

The following statements are valid as well.

5s. Every standard word has the only alignment of brackets such that the appeared nonassociative word is standard (the Shirshov theorem [40]).

6s. The factors $v, w$ of the nonassociative decomposition $[u]=[[v][w]]$ are the standard words such that $u=v w$ and $v$ has the minimal length $([41])$. 
Definition 4.2. A super-letter is a polynomial that equals a nonassociative standard word where the brackets mean (2). A super-word is a word in super-letters.

By 5 s every standard word $u$ defines the only super-letter, in what follows we will denote it by $[u]$. For example, the words $x_{1} x_{2}^{2}, x_{2}^{3} x_{3}, x_{1} x_{2} x_{3} x_{2}$, $x_{2} x_{3} x_{2} x_{3} x_{4}, x_{1} x_{2} x_{3}^{2} x_{2}$ are standard and they define the following superletters

$$
\begin{gathered}
{\left[x_{1} x_{2}^{2}\right]=\left[\left[x_{1} x_{2}\right] x_{2}\right], \quad\left[x_{2}^{3} x_{3}\right]=\left[x_{2}\left[x_{2}\left[x_{2} x_{3}\right]\right]\right], \quad\left[x_{1} x_{2} x_{3} x_{2}\right]=\left[\left[x_{1}\left[x_{2} x_{3}\right]\right] x_{2}\right],} \\
{\left[x_{2} x_{3} x_{2} x_{3} x_{4}\right]=\left[\left[x_{2} x_{3}\right]\left[x_{2}\left[x_{3} x_{4}\right]\right]\right], \quad\left[x_{1} x_{2} x_{3}^{2} x_{2}\right]=\left[\left[x_{1}\left[\left[x_{2} x_{3}\right] x_{3}\right]\right] x_{2}\right] .}
\end{gathered}
$$

In Theorem 2.4 we have $W=\alpha\left[x_{1} x_{2}^{n}\right]$. If the variables are ordered in the opposite way, $x_{2}>x_{1}$, then $x_{1} x_{2}^{n}$ is not a standard word, while $x_{2}^{n} x_{1}$ is, and one may see that $\left[\ldots\left[\left[x_{1} x_{2}\right] x_{2}\right] \ldots x_{2}\right]=\left(-p_{12}\right)^{n} p_{22}^{\frac{n(n-1)}{2}}\left[x_{2}^{n} x_{1}\right]$ provided that one of the existence conditions is valid (see Corollary 4.10 below). Therefore the quantified relations (11) can be written in a form of equality to zero of some super-letters:

$$
\left[x_{j} x_{i}^{n_{i j}}\right]=0, \quad\left[x_{j}^{n_{j i}} x_{i}\right]=0, \quad j<i .
$$

Let $D$ be a linearly ordered Abelian additive group. Suppose that some positive $D$-degrees $d_{1}, \ldots, d_{n} \in D$ are associated to $x_{1}, \ldots, x_{n}$. We define the degree of a word to be equal to $m_{1} d_{1}+\ldots+m_{n} d_{n}$ where $\left(m_{1}, \ldots, m_{n}\right)$ is the constitution of the word. The order and the degree on the super-letters are defined in the following way: $[u]>[v] \Longleftrightarrow u>v ; \mathrm{D}([u])=\mathrm{D}(u)$.

Definition 4.3. A super-letter $[u]$ is called hard in $H$ provided that its value in $H$ is not a linear combination of values of super-words of the same degree in less than $[u]$ super-letters and $G$-super-words of a lesser degree.

Definition 4.4. We say that a height of a super-letter $[u]$ of degree $d$ equals $h=h([u])$ if $h$ is the smallest number such that: First $p_{u u}$ is a primitive $t$-th root of unity and either $h=t$ or $h=t l^{r}$, where $l=\operatorname{char}(\mathbf{k})$; and then the value in $H$ of $[u]^{h}$ is a linear combination of super-words of degree $h d$ in less than $[u]$ super-letters and $G$-super-words of a lesser degree. If there exists no such number then the height equals infinity.

Clearly, if the algebra $H$ is $D$-homogeneous then one may omit the underlined parts of the above definitions.

Theorem 4.5 ([23, Theorem 2]). The set of all values in $H$ of all $G$-superwords $W$ in the hard super-letters $\left[u_{i}\right]$,

$$
W=g\left[u_{1}\right]^{n_{1}}\left[u_{2}\right]^{n_{2}} \cdots\left[u_{m}\right]^{n_{m}},
$$

where $g \in G, u_{1}<u_{2}<\ldots<u_{m}, n_{i}<h\left(\left[u_{i}\right]\right)$ is a basis of $H$. 
In order to find the set $U$ of PBW-generators it is necessary first to include in $U$ the values of all hard super-letters, then for each hard super-letter $[u]$ of a finite height, $h([u])=t l^{k}$, to add the values of $[u]^{t},[u]^{t l}, \ldots[u]^{t l^{(k-1)}}$, and next for each hard super-letter of infinite height such that $p_{u u}$ is a primitive $t$-th root of unity to add the value of $[u]^{t}$.

Obviously the set of PBW-generators plays the same role as the basis of the Lie algebra in the PBW theorem does. Nevertheless the $\mathbf{k}[G]$-bimodule generated by the PBW-generators is not uniquely defined. It depends on the ordering of the main generators, the $D$-degree, and under the action of antipode it transforms to a different bimodule of PBW-generators $\mathbf{k}[G] S(U)$.

Another way to construct PBW-generators is connected with the M. Kashiwara crystallization idea [20], [21]. M. Kashiwara considered the main parameter of the Drinfeld-Jimbo enveloping algebra as the temperature of some physical medium. When the temperature tends to zero the medium crystallizes. By this means a 'crystal' bases must appear. If we replace $p_{i j}$ with zero then $[u, v]$ turns into a monomial $u v$, while $[u]$ turns into a monomial $u$.

Lemma 4.6. Under the above monomial crystallization the set of $P B W$ generators constructed in Theorem 4.5 turns into another set of $P B W$ generators.

Proof. See [23, Corollary 1].

Lemma 4.7. A super-letter $[u]$ is hard in $H$ if and only if the value of $u$ is not a linear combination of values of lesser words of the same degree and $G$-words of a lesser degree.

Proof. See [23, Corollary 2].

Lemma 4.8. Let $B$ be a set of the super-letters containing $x_{1}, \ldots, x_{n}$. If each pair $[u],[v] \in B, u>v$ satisfies one of the following conditions:

1) $[[u][v]]$ is not a standard nonassociative word;

2) the super-letter $[[u][v]]$ is not hard in $H$;

3) $[[u][v]] \in B$,

then the set $B$ includes all hard in $H$ super-letters.

Proof. Let $[w]$ be a hard super-letter of minimal degree such that $[w] \notin B$. Then $[w]=[[u][v]], u>v$ where $[u],[v]$ are hard super-letters. Indeed, if $[u]$ is not hard then by Lemma 4.7 we have $u=\sum \alpha_{i} u_{i}+S$, where $u_{i}<u$ and $D\left(u_{i}\right)=D(u), D(S)<D(u)$. We have $u v=\sum \alpha_{i} u_{i} v+S v$, where $u_{i} v<u v$. Therefore by Lemma 4.7, the super-letter $[w]=[u v]$ can not be hard in $H$. Contradiction. Similarly, if $[v]$ is not hard then $v=\sum \alpha_{i} v_{i}+S, v_{i}<v$, $D\left(v_{i}\right)=D(v), D(S)<D(v)$. Therefore $u v=\sum \alpha_{i} u v_{i}+u S, u v_{i}<u v$, and again $[w]$ can not be hard. 
Thus, according to the choice of $[w]$, we get $[u],[v] \in B$. Since this pair satisfies neither condition 1) nor 2), the condition 3$),[u v] \in B$, holds.

Lemma 4.9. If $\mathbf{T} \in H$ is a skew primitive element then

$$
\mathbf{T}=\alpha[u]^{h}+\sum \alpha_{i} W_{i}+\sum \beta_{j} g_{j} W_{j}^{\prime}, \quad \alpha \neq 0,
$$

where $[u]$ is a hard super-letter, $W_{i}$ are basis super-words in super-letters less than $[u], D\left(W_{i}\right)=h D([u]), D\left(W_{j}^{\prime}\right)<h D([u])$. Here if $p_{u u}$ is not a root of unity then $h=1$; if $p_{u u}$ is a primitive $t$-th root of unity then $h=1$, or $h=t$, or $h=t l^{k}$, where $l$ is the characteristic.

Proof. Consider an expansion of $\mathbf{T}$ in terms of the basis (17)

$$
\mathbf{T}=\alpha g U+\sum_{i=1}^{k} \gamma_{i} g_{i} W_{i}+W^{\prime}, \quad \alpha \neq 0,
$$

where $g U, g_{i} W_{i}$ are different basis elements of maximal degree, and $U$ is one of the biggest words among $U, W_{i}$ with respect to the lexicographic ordering of words in the super-letters. On basis expansion of tensors, the element $\Delta(\mathbf{T})-\mathbf{T} \otimes 1-g_{t} \otimes \mathbf{T}$ has only one tensor of the form $g U \otimes \ldots$ and this tensor equals $g U \otimes \alpha(g-1)$. Therefore $g=1$ and one may apply [23, Lemma $13]$.

Corollary 4.10. If one of the existence conditions in Theorem 2.4 holds then

$$
\left[\ldots\left[\left[x_{1} x_{2}\right] x_{2}\right] \ldots x_{2}\right]=\left(-p_{12}\right)^{n} p_{22}^{\frac{n(n-1)}{2}}\left[x_{2}\left[x_{2} \ldots\left[x_{2} x_{1}\right] \ldots\right]\right] .
$$

Proof. Let us introduce the opposite order, $x_{2}>x_{1}$. Since $\left[\ldots\left[\left[x_{1} x_{2}\right] x_{2}\right] \ldots x_{2}\right]$ is a quantum Lie operation, it has a representation (18) where all addends have the same constitution, $(1, n)$. This implies $h=1, u=x_{2}^{n} x_{1}$. All standard words of the constitution less than or equal to $(1, n)$ are $x_{2}, x_{2}^{k} x_{1}, k \leq n$. By definition of the lexicographical order $x_{2}>x_{2}^{n} x_{1}$. Therefore $x_{2}$ does not occur in (18) as a super-letter. Since every addend has degree 1 in $x_{1}$, the equality (18) reduces to $\mathbf{T}=\alpha\left[x_{2}^{n} x_{1}\right]$. In order to find $\alpha$ one may to compare the coefficients at $x_{2}^{n} x_{1}$.

\section{Groebner-Shirshov relations systems.}

Let $x_{1}, \ldots, x_{n}$ be variables that have positive degrees $d_{1}, \ldots, d_{n} \in D$. Recall that a Hall ordering of words in $x_{1}, \ldots, x_{n}$ is an order when the words are compared firstly by the degree and then words of the same degree are compared by means of the lexicographic ordering. Consider a set of relations

$$
w_{i}=f_{i}, \quad i \in I,
$$

where $w_{i}$ is a word and $f_{i}$ is a linear combination of Hall lesser words. The system (20) is said to be closed under compositions or a Groebner-Shirshov 
relations system if first none of $w_{i}$ contains $w_{j}, i \neq j \in I$ as a sub-word, and then for each pair of words $w_{k}, w_{j}$ such that some nonempty terminal of $w_{k}$ coincides with an onset of $w_{j}$, that is $w_{k}=w_{k}^{\prime} v, w_{j}=v w_{j}^{\prime}$, the difference (a composition) $f_{k} w_{j}^{\prime}-w_{k}^{\prime} f_{j}$ can be reduced to zero in the free algebra by means of a sequence of one sided substitutions $w_{i} \rightarrow f_{i}, i \in I$.

Lemma 5.1 (Diamond Lemma [4], [6], [41]). If the system (20) is closed under compositions then the words that have none of $w_{i}$ as sub-words form a basis of the algebra $H$ defined by (20).

If none of the words $w_{i}$ has sub-words $w_{j}, j \neq i$, then the converse statement is valid as well. Indeed, any composition by means of substitutions $w_{i} \rightarrow f_{i}$ can be reduced to a linear combination of words that have no sub-words $w_{i}$. Since $f_{i} w_{j}^{\prime}-w_{i}^{\prime} f_{j}=\left(f_{i}-w_{i}\right) w_{j}^{\prime}-w_{i}^{\prime}\left(f_{j}-w_{j}\right)$, this linear combination equals zero in $H$. Therefore all the coefficients have to be zero.

Since Lemma 4.6 provides the basis that consists of words, the above note gives a way to construct the Groebner-Shirshov relations system for any quantum enveloping algebra.

Let $H$ be a character Hopf algebra generated by skew primitive semiinvariants $a_{1}, \ldots, a_{n}$ (or a braided bigraded Hopf algebra generated by grading homogeneous primitive elements $\left.a_{1}, \ldots, a_{n}\right)$ and let $x_{1}, \ldots, x_{n}$ be the related quantum variables. A non-hard in $H$ super-letter $[w]$ is referred to as a minimal one if first $w$ has no proper standard sub-words that define non-hard super-letters, and then $w$ has no sub-words $u^{h}$, where $[u]$ is a hard super-letter of the height $h$.

By Lemma 4.7, for every minimal non-hard in $H$ super-letter $[w]$ we may write a relation in $H$

$$
w=\sum \alpha_{i} w_{i}+\sum \beta_{j} g_{j} w_{j}
$$

where $w_{j}, w_{i}<w$ in the Hall sense, $D\left(w_{i}\right)=D(w), D\left(w_{j}\right)<D(w)$. In the same way if $[u]$ is a hard in $H$ super-letter of a finite height $h$ then

$$
u^{h}=\sum \alpha_{i} u_{i}+\sum \beta_{j} g_{j} u_{j},
$$

where $u_{j}, u_{i}<u^{h}$ in the Hall sense, $D\left(u_{i}\right)=h D(u), D\left(u_{j}\right)<h D(u)$. The relations (14) and the group operation provide the relations

$$
x_{i} g=\chi^{x_{i}}(g) g x_{i}, \quad g_{1} g_{2}=g_{3} .
$$

Theorem 5.2. The set of relations (21), (22), and (23) forms a GroebnerShirshov system that defines $H$. The basis determined by this system in Diamond Lemma coincides with the PBW basis obtained via monomial crystallization.

Proof. The property 4s implies that none of the left hand sides of (21), (22), (23) contains another one as a sub-word. Therefore by Lemma 4.6 it is 
sufficient to show that the set of all words $c$ determined in the Diamond Lemma coincides with the basis appeared in Lemma 4.6. By 3s we have $c=u_{1}^{n_{1}} u_{2}^{n_{2}} \cdots u_{k}^{n_{k}}$, where $u_{1}<\ldots<u_{k}$ is a sequence of standard words. Every word $u_{i}$ define a hard super-letter $\left[u_{i}\right]$ since in the opposite case $u_{i}$, and therefore $c$, contains a sub-word $w$ that defines a minimal non-hard super-letter $[w]$. In the same way $n_{i}$ does not exceed the height of $\left[u_{i}\right]$.

Lemma 5.3. In terms of Lemma 4.8 the set of all super-letters $[[u][v]]$ that satisfy the condition 2) contains all minimal non-hard super-letters, but nonhard generators $x_{i}$.

Proof. If $[w]$ is a minimal non-hard super-letter then $[w]=[[u][v]]$, where $[u],[v]$ are hard super-letters. By Lemma 4.8 we have $[u],[v] \in B$, while $[[u][v]]$ neither satisfies 1) nor 3).

\section{Quantification with constants.}

By means of the Diamond Lemma in some instances the investigation of a quantification with constants can be reduced to one of a simple quantification.

Let $H_{1}=G\left\langle x_{1}, \ldots, x_{k} \| F_{1}\right\rangle$ be a character Hopf algebra defined by the quantum variables $x_{1}, \ldots, x_{k}$ and the grading homogeneous relations $\{f=$ $\left.0: f \in F_{1}\right\}$, while $H_{2}=G\left\langle x_{k+1}, \ldots, x_{n} \| F_{2}\right\rangle$ is a character Hopf algebra defined by the quantum variables $x_{k+1}, \ldots, x_{n}$ and the grading homogeneous relations $\left\{h=0: h \in F_{2}\right\}$. Consider the algebra $H=G\left\langle x_{1}, \ldots, x_{n} \| F_{1}, F_{2}, F_{3}\right\rangle$, where $F_{3}$ is the following system of relations with constants

$$
\left[x_{i}, x_{j}\right]=\alpha_{i j}\left(1-g_{i} g_{j}\right), \quad 1 \leq i \leq k<j \leq n .
$$

If the conditions below are met then the character Hopf algebra structure on $H$ is uniquely determined:

$$
p_{i j} p_{j i}=1,1 \leq i \leq k<j \leq n ; \quad \chi^{x_{i}} \chi^{x_{j}} \neq 1 \Longrightarrow \alpha_{i j}=0 .
$$

Indeed, in this case the difference $w_{i j}$ between the left and right hand sides of (24) is a skew primitive semi-invariant of the free enveloping algebra $G\left\langle x_{1}, \ldots, x_{n}\right\rangle$. Consider the ideals of relations $I_{1}=\mathrm{id}\left(F_{1}\right)$ and $I_{2}=\mathrm{id}\left(F_{2}\right)$ of $H_{1}$ and $H_{2}$ respectively. They are, in the present context, Hopf ideals of $G\left\langle x_{1}, \ldots, x_{k}\right\rangle$ and $G\left\langle x_{k+1}, \ldots, x_{n}\right\rangle$, respectively. Therefore $V=I_{1}+I_{2}+$ $\sum \mathbf{k}[G] w_{i j}$ is an antipode stable coideal of $G\langle X\rangle$. Consequently the ideal generated by $V$ is a Hopf ideal. It remains to note that this ideal is generated in $G\langle X\rangle$ by $w_{i j}$ and $F_{1}, F_{2}$.

Lemma 6.1. Every hard in $H$ super-letter belongs to either $H_{1}$ or $H_{2}$, and it is hard in the related algebra.

Proof. If a standard word contains at least one of the letters $x_{i}, i \leq k$ then it has to start with one of them (see $2 \mathrm{~s}$ in $\S 4$ ). If this word contains a letter 
$x_{j}, j>k$ then it has a sub-word of the form $x_{i} x_{j}, i \leq k<j$. Therefore by Lemma 4.7 and relations (24) this word defines a non-hard super-letter.

The converse statement is not universally true. In order to formulate the necessary and sufficient conditions let us define partial skew derivatives:

$$
\begin{aligned}
& \partial_{i}\left(x_{j}\right)=\partial_{j}\left(x_{i}\right)=\alpha_{i j}\left(1-g_{i} g_{j}\right), \quad i \leq k<j \\
& \partial_{i}(v \cdot w)=\partial_{i}(v) \cdot w+p\left(x_{i}, v\right) v \cdot \partial_{i}(w), i \leq k, v, w \in \mathbf{k}\left\langle x_{k+1}, \ldots, x_{n}\right\rangle \\
& \partial_{j}(u \cdot v)=p\left(v, x_{j}\right) \partial_{j}(u) \cdot v+u \cdot \partial_{j}(v), j>k, u, v \in \mathbf{k}\left\langle x_{1}, \ldots, x_{k}\right\rangle
\end{aligned}
$$

Lemma 6.2. All hard in $H_{1}$ or $H_{2}$ super-letters are hard in $H$ if and only if $\partial_{i}(h)=0$ in $H_{2}$ for all $i \leq k, h \in F_{2}$, and $\partial_{j}(f)=0$ in $H_{1}$ for all $j>k$, $f \in F_{1}$. If these conditions are met then

$$
H \cong H_{2} \otimes_{\mathbf{k}[G]} H_{1}
$$

as $\mathbf{k}[G]$-bimodules, and the space generated by the skew primitive elements of $H$ equals the sum of these spaces for $H_{1}$ and $H_{2}$.

Proof. By (5) and (26) the following equalities are valid in $H$ :

$$
0=\left[x_{i}, h\right]=\partial_{i}(h) ; \quad 0=\left[f, x_{j}\right]=\partial_{j}(f), i \leq k<j .
$$

If all hard in $H_{1}$ or $H_{2}$ super-letters are hard in $H$ then $H_{1}, H_{2}$ are subalgebras of $H$. So (28) proves the necessity of the lemma conditions.

Conversely, let us consider an algebra $R$ defined by the generators $g \in G$, $x_{1}, \ldots, x_{n}$ and the relations (23), (24). Evidently this system is closed under the compositions. Therefore by Diamond Lemma the set of words $g v w$ forms a basis of $R$ where $g \in G$; $v$ is a word in $x_{j}, j>k$; and $w$ is a word in $x_{i}$, $i \leq k$. In other words $R$ as a bimodule over $\mathbf{k}[G]$ has a decomposition

$$
R=G\left\langle x_{k+1}, \ldots, x_{n}\right\rangle \otimes_{\mathbf{k}[G]} G\left\langle x_{1}, \ldots, x_{k}\right\rangle .
$$

Let us show that the two sided ideal of $R$ generated by $F_{2}$ coincides with the right ideal $I_{2} R=I_{2} \otimes_{\mathbf{k}[G]} G\left\langle x_{1}, \ldots, x_{k}\right\rangle$. It will suffice to show that $I_{2} R$ admits left multiplication by $x_{i}, i \leq k$. If $v$ is a word in $x_{k+1}, \ldots, x_{n}, h \in F_{2}$, $r \in R$ then $x_{i} v h r=\left[x_{i}, v h\right] r+p\left(x_{i}, v h\right) v h x_{i} r$. The second term belongs to $I_{2} R$, while the first one can be rewritten by $(5):\left[x_{i}, v\right] h+p\left(x_{i}, v\right) v\left[x_{i}, h\right]$. Both of these addends belong to $I_{2} R$ since $\left[x_{i}, v\right]=\partial_{i}(v) \in G\left\langle x_{k+1}, \ldots, x_{n}\right\rangle$ and $\left[x_{i}, h\right]=\partial_{i}(h) \in I_{2}$.

Furthermore, consider a quotient algebra $R_{1}=R / I_{2} R$ :

$$
\begin{aligned}
R_{1} & =\left(G\left\langle x_{k+1}, \ldots, x_{n}\right\rangle \otimes_{\mathbf{k}[G]} G\left\langle x_{1}, \ldots, x_{k}\right\rangle\right) /\left(I_{2} \otimes_{\mathbf{k}[G]} G\left\langle x_{1}, \ldots, x_{k}\right\rangle\right) \\
& =H_{2} \otimes_{\mathbf{k}[G]} G\left\langle x_{1}, \ldots, x_{k}\right\rangle,
\end{aligned}
$$

where the equality means the natural isomorphism of $\mathbf{k}[G]$-bimodules.

Along similar lines, the left ideal $R_{1} I_{1}=H_{2} \otimes_{\mathbf{k}[G]} I_{1}$ of this quotient algebra coincides with the two-sided ideal generated by $F_{1}$. Therefore

$$
H=R_{1} / R_{1} I_{1}=H_{2} \otimes_{\mathbf{k}[G]} G\left\langle x_{1}, \ldots, x_{k}\right\rangle / H_{2} \otimes_{\mathbf{k}[G]} I_{1}=H_{2} \otimes_{\mathbf{k}[G]} H_{1} .
$$


Thus the monotonous restricted $G$-words in hard in $H_{1}$ or $H_{2}$ super-letters form a basis of $H$. This, in particular, proves the first statement.

Now let $T=\sum \alpha_{t} g_{t} V_{t} W_{t}$ be the basis decomposition of a skew primitive element, $g_{t} \in G, V_{t} \in H_{2}, W_{t} \in H_{1}, \alpha_{t} \neq 0$. We have to show that for each $t$ one of the super-words $V_{t}$ or $W_{t}$ is empty. Suppose that it is not so. Among the addends with nonempty $V_{t}, W_{t}$ we choose the largest one in the Hall sense, say $g_{s} V_{s} W_{s}$. Under the basis decomposition of $\Delta(T)-T \otimes$ $1-g(T) \otimes T$ the term $\alpha_{s} g_{s} g\left(V_{s}\right) W_{s} \otimes g_{s} V_{s}$ appears and cannot be canceled with other. Indeed, since the coproduct is homogeneous (see [23, Lemma $9]$ ) and since under the basis decomposition the super-words are decreased (see [23, Lemma 7]) the product $\alpha_{s}\left(g_{s} \otimes g_{s}\right) \Delta\left(V_{s}\right) \Delta\left(W_{s}\right)$ has the only term of the above type. By the same reasons $\alpha_{t}\left(g_{t} \otimes g_{t}\right) \Delta\left(V_{t}\right) \Delta\left(W_{t}\right)$ has a term of the above type only if $V_{t} \geq V_{s}$ and $W_{t} \geq W_{s}$ with respect to the Hall ordering of the set of all super-words. However, by the choice of $s$, we have $D\left(V_{s} W_{s}\right) \geq D\left(V_{t} W_{t}\right)$. Hence $D\left(V_{t}\right)=D\left(V_{s}\right)$ and $D\left(W_{t}\right)=D\left(W_{s}\right)$. In particular $V_{t}$ is not a proper onset of $V_{s}$. Therefore $V_{t}=V_{s}$ since otherwise the inequality $V_{t}>V_{s}$ yields a contradiction $V_{t} W_{t}>V_{s} W_{s}$. The inequality $W_{t}>W_{s}$ yields the same contradiction. Therefore $V_{t}=V_{s}$ and $W_{t}=W_{s}$, in which case $g_{t} g\left(V_{t}\right) W_{t} \otimes g_{t} V_{t}=g_{s} g\left(V_{s}\right) W_{s} \otimes g_{s} V_{s}$. Thus $g_{t}=g_{s}$ and $t=s$.

\section{Quantification of the classical series.}

In this section we apply the above general results to the infinite series $A_{n}$, $B_{n}, C_{n}, D_{n}$ of nilpotent Lie algebras defined by the Serre relations (11) or, equivalently, (16). Let $\mathfrak{g}$ be any such Lie algebra.

Lemma 7.1. If a standard word $u$ has no sub-words of the type

$$
x_{i}^{s} x_{j} x_{i}^{m}, \text { where } s+m=1-a_{i j}
$$

then $[u]$ is a hard in $U_{P}(\mathfrak{g})$ super-letter.

Proof. Let $R$ be defined by the generators $x_{1}, \ldots, x_{n}$ and the relations

$$
x_{i}^{s} x_{j} x_{i}^{m}=0, \text { where } s+m=1-a_{i j} .
$$

Clearly (31) implies (16). Therefore $R$ is a homomorphic image of $U_{P}^{b}(\mathfrak{g})$. The system (31) is closed under compositions since a composition of monomial relations always has the form $0=0$.

Let $u$ have no sub-words (30). Then the value of $u$ in $R$ belongs to the basis of $R$ defined in Diamond Lemma. If $[u]$ is not hard then, by the homogeneous version of Lemma 4.7, $u$ is a linear combination of lesser words in $U_{P}^{b}(\mathfrak{g})$. Therefore $u$ is a linear combination of lesser words in $R$ as well. This contradicts the fact that $u$ belongs to the basis of $R$ defined in Diamond Lemma. 
Theorem $\mathbf{A}_{\boldsymbol{n}}$. Suppose that $\mathfrak{g}$ is of the type $A_{n}$, and $p_{i i} \neq-1$. Denote by $B$ the set of the super-letters given below:

$$
\left[u_{k m}\right] \stackrel{d f}{=}\left[x_{k} x_{k+1} \ldots x_{m}\right], \quad 1 \leq k \leq m \leq n .
$$

The following statements are valid.

1. The values of $\left[u_{k m}\right]$ in $U_{P}(\mathfrak{g})$ form a $P B W$-generators set.

2. Each of the super-letters (32) has infinite height in $U_{P}(\mathfrak{g})$.

3. The values of all non-hard in $U_{P}(\mathfrak{g})$ super-letters equal zero.

4. The following relations with (23) form the Groebner-Shirshov relations system for $U_{P}(\mathfrak{g})$ :

$$
\begin{array}{ll}
{\left[u_{0}\right] \stackrel{d f}{=}\left[x_{k} x_{m}\right]=0,} & 1 \leq k<m-1<n ; \\
{\left[u_{1}\right] \stackrel{d f}{=}\left[x_{k} x_{k+1} \ldots x_{m} x_{k+1}\right]=0,} & 1 \leq k<m \leq n ; \\
{\left[u_{2}\right] \stackrel{d f}{=}\left[x_{k} x_{k+1} \ldots x_{m} x_{k} x_{k+1} \ldots x_{m+1}\right]=0,} & 1 \leq k \leq m<n .
\end{array}
$$

5. If $p_{11} \neq 1$ then the generators $x_{i}$, the constants $1-g, g \in G$, and, in the case that $p_{11}$ is a primitive $t$-th root of 1 , the elements $x_{i}^{t}, x_{i}^{t l^{k}}$ form a basis of the space $\mathfrak{g}_{P}=L\left(U_{P}(\mathfrak{g})\right)$ generated by skew primitive elements. Here $l$ is the characteristic of the ground field.

6. If $p_{11}=1$ then the elements (32) and, in the case $l>0$, their $l^{k}$-th powers, together with $1-g, g \in G$ form a basis of $\mathfrak{g}_{P}$.

By Corollary 2.5 the relations (11) with a Cartan matrix $A$ of type $A_{n}$ admit a quantification if and only if

$$
p_{i i}=p_{11}, \quad p_{i i+1} p_{i+1 i}=p_{11}^{-1} ; \quad p_{i j} p_{j i}=1, i-j>1 .
$$

In this case the quantified relations (16) take up the form

$$
\begin{aligned}
& x_{i} x_{i+1}^{2}=p_{i i+1}\left(1+p_{i+1 i+1}\right) x_{i+1} x_{i} x_{i+1}-p_{i i+1}^{2} p_{i+1 i+1} x_{i+1}^{2} x_{i}, \\
& x_{i}^{2} x_{i+1}=p_{i i+1}\left(1+p_{i i}\right) x_{i} x_{i+1} x_{i}-p_{i i+1}^{2} p_{i i} x_{i+1} x_{i}^{2}, \\
& x_{i} x_{j}=p_{i j} x_{j} x_{i}, \quad i-j>1 .
\end{aligned}
$$

Definition 7.2. We introduce the congruence $u \equiv_{k} v$ on $G\langle X\rangle$. This congruence means that the value of $u-v$ in $U_{P}^{b}(\mathfrak{g})$ belongs to the subspace generated by values of all words with the initial letters $x_{i}, i \geq k$.

Clearly, this congruence admits right multiplication by arbitrary polynomials as well as left multiplication by the independent of $x_{k-1}$ ones (see (37)). For example, by (35) and (36) we have

$$
x_{i} x_{i+1}^{2} \equiv_{i+1} 0 ; \quad x_{i} x_{i+1} x_{i} \equiv_{i+1} \alpha x_{i}^{2} x_{i+1}, \quad \alpha \neq 0 .
$$

Lemma 7.3. If $y=x_{i}, m+1 \neq i>k$ or $y=x_{i}^{2}, m+1=i>k$ then

$$
u_{k m} y \equiv_{k+1} 0 \text {. }
$$


Proof. Let $y=x_{m+1}^{2}, m+1>k$. By (38) and (37) we have that $u_{k m} y=$ $u_{k m-1} x_{m} x_{m+1}^{2} \equiv_{m+1} 0$. If $y=x_{i}$ and $m+1 \neq i>k$ then we get $u_{k m} y=$ $\alpha u_{k i-1} \underline{x_{i} x_{i+1} x_{i}} u_{i+2 m} \equiv_{i+1} \beta \underline{u_{k i-1} x_{i}^{2}} u_{i+1 m} \equiv_{k+1} 0$ by the above case.

Lemma 7.4. The brackets in $\left[u_{k m}\right]$ are left-ordered, $\left[u_{k m}\right]=\left[x_{k}\left[u_{k+1 m}\right]\right]$.

Proof. The statement immediately follows from the properties $6 \mathrm{~s}$ and $2 \mathrm{~s}$.

Lemma 7.5. If a nonassociative word $\left[\left[u_{k m}\right]\left[u_{r s}\right]\right]$ is standard then $k=m \leq$ $r$; or $r=k+1, m \geq s ;$ or $r=k, m<s$.

Proof. By definition, $u_{k m}>u_{r s}$ if and only if either $k<r$; or $k=r, m<s$. If $k=m$ then $u_{k m}=x_{k}$ and $m \leq r$. If $k \neq m$ then $\left[u_{k m}\right]=\left[x_{k}\left[u_{k+1 m}\right]\right]$. Therefore $u_{k+1 m} \leq u_{r s}$, i.e., either $k+1>r$; or $k+1=r$ and $m \geq s$. The former case contradicts $k<r$ while the latter one does $k=r$. Thus only the possibilities set in the lemma remain.

Lemma 7.6. If $[w]=\left[\left[u_{k m}\right]\left[u_{r s}\right]\right], n \geq 1$ is a standard nonassociative word then the constitution of $[w]^{h}$ does not equal the constitution of any superword in less than $[w]$ super-letters from $B$.

Proof. The inequalities at the last column of the following tableaux are valid for all $[u] \in B$ that are less than the super-letters located on the same row, where as above $\operatorname{deg}_{i}(u)$ means the degree of $u$ in $x_{i}$.

$$
\begin{array}{lll}
{\left[x_{k} u_{k+1 s}\right]} & & \operatorname{deg}_{k}(u) \leq \operatorname{deg}_{s+1}(u) ; \\
{\left[x_{k} u_{r s}\right],} & k \leq r \neq k+1 & \operatorname{deg}_{k}(u) \leq \operatorname{deg}_{k+1}(u) ; \\
{\left[u_{k m} u_{k+1 s}\right],} & m \geq s & \operatorname{deg}_{k}(u) \leq \operatorname{deg}_{m+1}(u) ; \\
{\left[u_{k m} u_{k s}\right],} & m<s & \operatorname{deg}_{k}(u) \leq \operatorname{deg}_{m+1}(u) .
\end{array}
$$

If all super-letters of a super-word $U$ satisfy one of these inequalities then $U$ does as well. Clearly, no one of the super-letters in the first column satisfies the degree inequality on the same row. Finally, by Lemma 7.5 the first column contains all standard nonassociative words of the type $\left[\left[u_{k m}\right]\left[u_{r s}\right]\right]$.

Lemma 7.7. If $p_{11} \neq 1$ then the values of $\left[u_{k m}\right]^{h}, k<m, h \geq 1$ are not skew primitive, in particular they are nonzero.

Proof. The sub-algebra generated by $x_{2}, \ldots x_{n}$ is defined by the Cartan matrix of the type $A_{n-1}$. This allows us to use induction on $n$. If $n=1$ then the lemma is correct in the sense that $\left[u_{k m}\right]^{h}=x_{1}^{h} \neq 0$.

Let $n>1$. If $k>1$ then we may use the inductive supposition directly. Consider the decomposition $\Delta\left(\left[u_{1 m}\right]\right)=\sum u^{(1)} \otimes u^{(2)}$. Since

$$
\left[u_{1 m}\right]=x_{1}\left[u_{2 m}\right]-p\left(x_{1}, u_{2 m}\right)\left[u_{2 m}\right] x_{1},
$$

we have 


$$
\begin{aligned}
\Delta\left(\left[u_{1 m}\right]\right)= & \left(x_{1} \otimes 1+g_{1} \otimes x_{1}\right) \Delta\left(\left[u_{2 m}\right]\right) \\
& -p\left(x_{1}, u_{2 m}\right) \Delta\left(\left[u_{2 m}\right]\right)\left(x_{1} \otimes 1+g_{1} \otimes x_{1}\right) .
\end{aligned}
$$

Therefore the sum of all tensors $u^{(1)} \otimes u^{(2)}$ with $\operatorname{deg}_{1}\left(u^{(2)}\right)=1, \operatorname{deg}_{k}\left(u^{(2)}\right)=$ $0, k>1$ has the form $\varepsilon g_{1}\left[u_{2 m}\right] \otimes x_{1}$, where $\varepsilon=1-p\left(x_{1}, u_{2 m}\right) p\left(u_{2 m}, x_{1}\right)$ since $\left[u_{2 m}\right] g_{1}=p\left(u_{2 m}, x_{1}\right) g_{1}\left[u_{2 m}\right]$. By (34) we have $p_{i j} p_{j i}=1$ for $i-1>j$. Therefore $\varepsilon=1-p_{12} p_{21}=1-p_{11}^{-1} \neq 0$.

This implies that in the decomposition $\Delta\left(\left[u_{1 m}\right]^{h}\right)=\sum v^{(1)} \otimes v^{(2)}$ the sum of all tensors $v^{(1)} \otimes v^{(2)}$ with $\operatorname{deg}_{1}\left(v^{(2)}\right)=h, \operatorname{deg}_{k}\left(v^{(2)}\right)=0, k>1$ equals $\varepsilon^{h}\left[u_{2 m}\right]^{h} \otimes x_{1}^{h}$. Thus $\left[u_{1 m}\right]^{h}$ is not skew primitive in $U_{P}(\mathfrak{g})$.

Proof of Theorem $\mathrm{A}_{n}$. Let us show firstly that $B$ satisfies the conditions of Lemma 4.8. By Lemma $4.7[w]=\left[\left[u_{k m}\right]\left[u_{r s}\right]\right]$ is non-hard if the value of $u_{k m} u_{r s}$ is a linear combination of lesser words. For $k=m, r=k+1$ we have $[w]=\left[u_{k s}\right] \in B$. If $k=m, r>k+1$ then the word $x_{k} u_{r s}$ can be diminished by (36) or (37). If $k \neq m$ then by Lemma 7.5 the word $u_{k m} u_{r s}$ has a sub-word of the type $u_{1}$ or $u_{2}$. Thus we need show only that the values in $U_{P}(\mathfrak{g})$ of $u_{1}$ and $u_{2}$ are linear combinations of lesser words.

The word $u_{1}$ has such a representation by Lemma 7.3. Consider the word $u_{2}$. Let us show by downward induction on $k$ that

$$
u_{k m} u_{k m+1} \equiv_{k+1} \gamma u_{k m+1} u_{k m}, \quad \gamma \neq 0 .
$$

If $k=m$ then one may use (36) with $i=k$. Let $k<m$. Let us transpose the second letter $x_{k}$ of $u_{2}$ as far to the left as possible by (37). We get

$$
u_{2}=\alpha \underline{x_{k} x_{k+1} x_{k}} x_{k+2} \cdots x_{m} x_{k+1} \cdots x_{m+1}, \quad \alpha \neq 0 .
$$

By (36) we have

$$
u_{2} \equiv_{k+1} \beta x_{k}^{2}\left(x_{k+1} x_{k+2} \cdots x_{m} x_{k+1} \cdots x_{m+1}\right), \quad \beta \neq 0 .
$$

Let us apply the inductive supposition to the word in the parentheses. Since $x_{i}, i>k+1$ commutes with $x_{k}^{2}$ according to the formulae (37), we get

$$
u_{2} \equiv_{k+1} \gamma \underline{x_{k}^{2} x_{k+1}} x_{k+2} \cdots x_{m+1} x_{k+1} \cdots x_{m} .
$$

Now it remains to replace the underlined sub-word according to (36) and then to transpose the second letter $x_{k}$ to its former position by (37).

(Note. For the diminishing of $u_{1}, u_{2}$ we did not use, and we could not use, the relation $\left[x_{n-1} x_{n}^{2}\right]=0$ since $\operatorname{deg}_{n}\left(u_{1}\right) \leq 1, \operatorname{deg}_{n}\left(u_{2}\right) \leq 1$.)

Thus $B$ satisfies the conditions of Lemma 4.8. Since none of $\left[u_{k m}\right]$ has sub-words (30), Lemmas 7.1 and 4.8 show that the first statement is correct.

If $\left[u_{k m}\right]$ has a finite height $h$ then the value of the polynomial $\left[u_{k m}\right]^{h}$ in $U_{P}(\mathfrak{g})$ is a linear combination of words in hard super-letters that are less than $\left[u_{k m}\right]$. However by Lemma 7.6 this linear combination is trivial, 
$\left[u_{k m}\right]^{h}=0$, since the defining relations are homogeneous. By Lemma 7.7 the second statement is correct for $p_{11} \neq 1$.

Similarly consider the skew primitive elements. Since both the defining relations and the coproduct are homogeneous, all the homogeneous components of a skew primitive element are skew primitive itself. Therefore it remains to describe all skew primitive elements homogeneous in each $x_{i}$. Let $T$ be such an element. By Lemma 4.9 we have

$$
T=[u]^{h}+\sum \alpha_{i} W_{i},
$$

where $[u]$ is a hard super-letter, $u=u_{k m}$, and $W_{i}$ are super-words in less than $[u]$ super-letters from $B$. By the homogeneity all $W_{i}$ have the same constitution as $\left[u_{k m}\right]^{h}$ does. However by Lemma 7.6 there exist no such super-words. This means that the only possible case is $T=\left[u_{k m}\right]^{h}$. Thus, by Lemma 7.7 the fifth statement is valid as well.

If $p_{11}=1$ then $p_{i j} p_{j i}=p_{i i}=1$ for all $i, j$. So we are under the conditions of Example 1, that is $U_{P}^{b}(\mathfrak{g})$ is the universal enveloping algebra of the color Lie algebra $\mathfrak{g}^{\text {col }}$. Further, $\left[u_{k m}\right] \in \mathfrak{g}^{\text {col }}$ and $\left[u_{k m}\right]$ are linearly independent in $\mathfrak{g}^{\text {col }}$ since they are hard super-letters and no one of them can be a linear combination of the lesser ones. Let us complete $B$ to a homogeneous basis $B^{\prime}$ of $\mathfrak{g}^{\text {col }}$. Then by the PBW theorem for the color Lie algebras the products $b_{1}^{n_{1}} \cdots b_{k}^{n_{k}}, b_{1}<\ldots<b_{k}$ form a basis of $U\left(\mathfrak{g}^{\mathrm{col}}\right)=U_{P}^{b}(\mathfrak{g})$. However, the monotonous restricted words in $B$ form a basis of $U_{P}^{b}(\mathfrak{g})$ also. Thus $B^{\prime}=B$ and all hard super-letters have the infinite height.

In particular, we get that the second statement is valid in complete extent. Moreover, if $p_{11}=1$ then $p\left(u_{k m}, u_{k m}\right)=1$, thus for $l=0$ all homogeneous skew primitive elements became exhausted by $\left[u_{k m}\right]$, while for $l>0$ the powers $\left[u_{k m}\right]^{k}$ are added to them (of course, here $l \neq 2$ since $-1 \neq p_{i i}=1$ ).

So we have proved all statements, but the third and fourth ones. These statements will follow Theorem 5.2 and Lemma 5.3 if we prove that all non-hard super-letters $\left[\left[u_{k m}\right]\left[u_{r s}\right]\right]$ equal zero in $U_{P}(\mathfrak{g})$. By the homogeneous definition, $\left[\left[u_{k m}\right]\left[u_{r s}\right]\right]$ is a linear combination of super-words in lesser hard super-letters. However, by Lemma 7.6, there exist no such super-words of the same constitution. Therefore, by the homogeneity, the above linear combination equals zero.

Theorem $\mathbf{B}_{n}$. Let $\mathfrak{g}$ be of the type $B_{n}$, and $p_{i i} \neq-1,1 \leq i<n, p_{n n}^{[3]}$ $\stackrel{d f}{=} p_{n n}^{2}+p_{n n}+1 \neq 0$. Denote by $B$ the set of the super-letters given below:

$$
\begin{array}{ll}
{\left[u_{k m}\right] \stackrel{d f}{=}\left[x_{k} x_{k+1} \ldots x_{m}\right],} & 1 \leq k \leq m \leq n ; \\
{\left[w_{k m}\right] \stackrel{d f}{=}\left[x_{k} x_{k+1} \ldots x_{n} \cdot x_{n} x_{n-1} \ldots x_{m}\right],} & 1 \leq k<m \leq n .
\end{array}
$$

The following statements are valid.

1. The values of (44) in $U_{P}(\mathfrak{g})$ form the $P B W$-generators set. 
2. Every super-letter $[u] \in B$ has infinite height in $U_{P}(\mathfrak{g})$.

3. The relations (23) with the following ones form a Groebner-Shirshov system for $U_{P}(\mathfrak{g})$.

$$
\begin{array}{ll}
{\left[u_{0}\right] \stackrel{d f}{=}\left[x_{k} x_{m}\right]=0,} & 1 \leq k<m-1<n ; \\
{\left[u_{1}\right] \stackrel{d f}{=}\left[u_{k m} x_{k+1}\right]=0,} & 1 \leq k<m \leq n, k \neq n-1 ; \\
{\left[u_{2}\right] \stackrel{d f}{=}\left[u_{k m} u_{k m+1}\right]=0,} & 1 \leq k \leq m<n ; \\
{\left[u_{3}\right] \stackrel{d f}{=}\left[w_{k m} x_{k+1}\right]=0,} & 1 \leq k<m \leq n, k \neq m-2 ; \\
{\left[u_{4}\right] \stackrel{d f}{=}\left[w_{k k+1} x_{k+2}\right]=0,} & 1 \leq k<n-1 ; \\
{\left[u_{5}\right] \stackrel{d f}{=}\left[w_{k m} w_{k m-1}\right]=0,} & 1 \leq k<m-1 \leq n-1 ; \\
{\left[u_{6}\right] \stackrel{d f}{=}\left[u_{k n}^{2} x_{n}\right]=0,} & 1 \leq k<n .
\end{array}
$$

4. If $p_{11} \neq 1$ then the generators $x_{i}$ and their powers $x_{i}^{t}, x_{i}^{t t^{k}}$, such that $p_{i i}$ is a primitive $t$-th root of 1 , together with the constants $1-g, g \in G$ form a basis of $\mathfrak{g}_{P}=L\left(U_{P}(\mathfrak{g})\right)$. Here $l$ is the characteristic of the ground field.

5. If $p_{n n}=p_{11}=1$ then the elements (44) and, for $l>0$, their $l^{k}$-th powers, together with $1-g, g \in G$ form a basis of $\mathfrak{g}_{P}$. If $p_{n n}=-p_{11}=$ -1 then $\left[u_{k n}\right]^{2},\left[u_{k n}\right]^{2 l^{k}}$ are added to them.

Recall that in the case $B_{n}$ the algebra $U_{P}^{b}(\mathfrak{g})$ is defined by (35), (36), (37) where in (35) the last relation, $i=n-1$, is replaced with

$$
x_{n-1} x_{n}^{3}=p_{n-1 n} p_{n n}^{[3]} x_{n} x_{n-1} x_{n}^{2}-p_{n-1 n}^{2} p_{n n} p_{n n}^{[3]} x_{n}^{2} x_{n-1} x_{n}+p_{n-1 n}^{3} p_{n n}^{3} x_{n}^{3} x_{n-1} \text {. }
$$

By Corollary 2.5 we get the existence conditions

$$
p_{i i}=p_{11}, p_{i i+1} p_{i+1 i}=p_{11}^{-1}=p_{n n}^{-2}, 1 \leq i \leq n-1 ; p_{i j} p_{j i}=1, i-j>1 .
$$

The relations (35) and (46) show that

$$
x_{i} x_{i+1}^{2} \equiv_{i+1} 0, \quad i<n-1 ; \quad x_{n-1} x_{n}^{3} \equiv_{n} 0,
$$

while the relations (36) imply

$$
x_{i} x_{i+1} x_{i} \equiv_{i+1} \alpha x_{i}^{2} x_{i+1}, \quad \alpha \neq 0 .
$$

By means of these relations and (37), (46) we have

$$
x_{n-2} x_{n-1}{\underline{x_{n}^{2}} x_{n-1} x_{n}}_{\equiv_{n-1} 0 .}
$$

Lemma 7.8. The brackets in $\left[w_{k m}\right]$ are set by the recurrence formulae:

$$
\begin{array}{lc}
{\left[w_{k m}\right]=\left[x_{k}\left[w_{k+1 m}\right]\right],} & \text { if } 1 \leq k<m-1<n ; \\
{\left[w_{k k+1}\right]=\left[\left[w_{k k+2}\right] x_{k+1}\right],} & \text { if } 1 \leq k<n .
\end{array}
$$

Here by the definition $w_{k n+1}=u_{k n}$. 
Proof. It is enough to use the property $6 \mathrm{~s}$ and then $1 \mathrm{~s}$ and $2 \mathrm{~s}$.

Lemma 7.9. The nonassociative word $\left[\left[w_{k m}\right]\left[w_{r s}\right]\right]$ is standard only in the following two cases: 1) $s \geq m>k+1=r$; 2) $s<m, r=k$.

Proof. If $\left[\left[w_{k m}\right]\left[w_{r s}\right]\right]$ is standard then $w_{k m}>w_{r s}$ and by (51) either $w_{k+1} \leq$ $w_{r s}$, or $m=k+1$ and $x_{k+1} \leq w_{r s}$. The inequality $w_{k m}>w_{r s}$ is correct only in two cases: $k<r$ or $k=r, m>s$. We get four possibilities:

1) $k<r, \quad k<m-1, \quad w_{k+1 m} \leq w_{r s}$;

2) $k<r, m=k+1, x_{k+1} \leq w_{r s}$;

3) $k=r, m>s, k<m-1, w_{k+1 m} \leq w_{r s}$;

4) $k=r, m>s, m=k+1, x_{k+1} \leq w_{r s}$.

Only the first and third ones are consistent since in the second case $x_{k+1} \leq$ $w_{r s}$ implies $k+1>r$, while in the fourth case $r<s$ and $k=r<s<m=$ $k+1$. If now we decode $w_{k+1 m} \leq w_{r s}$ in the first and third cases, we get the two possibilities mentioned in the lemma.

Lemma 7.10. The nonassociative word $\left[\left[u_{k m}\right]\left[w_{r s}\right]\right]$ is standard only in the following two cases: 1) $k=r$; 2) $k=m<r$.

Proof. The inequality $u_{k m}>w_{r s}$ means $k \leq r$. Since $\left[u_{k m}\right]=\left[x_{k}\left[u_{k+1 m}\right]\right]$, for $k \neq m$ we get $u_{k+1 m} \leq w_{r s}$, so $k+1>r$ and $k=r$. If $k=m \neq r$ then $x_{m}>w_{r s}$ and $m<r$.

Lemma 7.11. The nonassociative word $\left[\left[w_{k m}\right]\left[u_{r s}\right]\right]$ is standard only in the following two cases: 1) $r=k+1<m$;2) $r=k+1=m=s$.

Proof. The inequality $w_{k m}>u_{r s}$ implies $r>k$. If $k<m-1$ then by the first formula (51) we have $w_{k+1 m} \leq u_{r s}$ that is equivalent to $k+1 \geq r$. Therefore $r=k+1<m$. If $k=m-1$ then by the second formula (51) we get $x_{k+1} \leq u_{r s}$, i.e., either $k+1>r$ or $k+1=r=s$. The former case contradicts $r>k$ while the latter one is mentioned in the lemma.

Lemma 7.12. If $[u],[v] \in B$ then one of the statements below is correct.

1) $[[u][v]]$ is not a standard nonassociative word;

2) $u v$ contains a sub-word of one of the types $u_{0}, u_{1}, u_{2}, u_{3}, u_{4}, u_{5}, u_{6}$;

3) $[[u][v]] \in B$.

Proof. The proof results from Lemmas 7.5, 7.9, 7.10, 7.11.

Lemma 7.13. If a super-word $W$ equals one of the super-letters $\left[u_{1}\right]-\left[u_{6}\right]$ or $\left[u_{k m}\right]^{h},\left[w_{k m}\right]^{h}, h \geq 1$ then its constitution does not equal the constitution of any super-word in less than $W$ super-letters from $B$.

Proof. The proof is akin to Lemma 7.6 with the following tableaux:

$$
\begin{aligned}
& {\left[u_{k m}\right], \quad\left[u_{k m} x_{k+1}\right], \quad\left[u_{k m} u_{k m+1}\right]} \\
& {\left[w_{k m}\right],\left[w_{k m} x_{k+1}\right],\left[w_{k m} w_{k m-1}\right]} \\
& {\left[w_{k k+1} x_{k+2}\right]} \\
& {\left[u_{k n}^{2} x_{n}\right]}
\end{aligned}
$$$$
\operatorname{deg}_{k}(u) \leq \operatorname{deg}_{m+1}(u) \text {; }
$$$$
2 \operatorname{deg}_{k}(u) \leq \operatorname{deg}_{m-1}(u) \text {; }
$$$$
\operatorname{deg}_{k}(u)=0 \text {; }
$$$$
\operatorname{deg}_{k}(u) \leq \operatorname{deg}_{n}(u) \text {. }
$$ 
Lemma 7.14. If $y=x_{i}, m-1 \neq i>k$ or $y=x_{i}^{2}, m-1=i>k$ then

$$
w_{k m} y \equiv_{k+1} 0 \text {. }
$$

Proof. If $i<m-1$ then by means of (37) it is possible to permute $y$ to the left beyond $x_{n}^{2}$ and use Lemma 7.3 with $m^{\prime}=n-1$. If $y=x_{i}^{2}, m-1=i>k$ then by the above case, $i<m-1$, we get

$$
w_{k m} y=w_{k m+1} \underline{x_{m} x_{m-1}^{2}}=\underline{w_{k m+1} x_{m-1}}\left(\alpha x_{m} x_{m-1}+\beta x_{m-1} x_{m}\right) \equiv_{k+1} 0,
$$

where for $m=n$ by definition $w_{k n+1}=u_{k n}$, and $u_{k n} x_{n-1} \equiv_{n-1} 0$.

If $y=x_{i}, i=m>k$ then for $m=n$ one may use the second equality (48). For $m<n$ we have $w_{k m} y=w_{k m+1} y_{1}$ where $y_{1}=x_{m}^{2}$. Therefore for $k<n-1$ we may use (54) with $m+1$ in place of $m$. For $k=n-1$ we have $w_{k m} x_{n}=x_{n-1} x_{n}^{3} \equiv_{n} 0$.

Finally, if $y=x_{i}, i>m>k$ then by (37) we have $w_{k m} y=\alpha w_{k i+1} x_{i} x_{i-1} x_{i} \cdot v$. For $i=n$ one may use (50), while for $i<n$, changing the underlined word according to (35), we may use the above considered cases: $m^{\prime}-1=i^{\prime}$, where $m^{\prime}=i+1, i^{\prime}=i$; and $i^{\prime}<m^{\prime}-1$, where $m^{\prime}=i+1, i^{\prime}=i-1$.

Another interesting relation appears if we multiply (46) by $x_{n-1}$ from the left and subtract (36) with $i=n-1$ multiplied from the right by $x_{n}^{2}$ :

$$
x_{n-1} x_{n} x_{n-1} x_{n}^{2} \equiv_{n} \alpha x_{n-1} x_{n}^{2} x_{n-1} x_{n},
$$

in which case $\alpha=p_{n-1 n} p_{n n}^{[3]} \neq 0$.

Lemma 7.15. For $k<s<m \leq n$ the following relation is valid.

$$
w_{k m} w_{k s} \equiv_{k+1} \varepsilon w_{k s} w_{k m}, \quad \varepsilon \neq 0 .
$$

Proof. Let us use downward induction on $k$. For this we first transpose the second letter $x_{k}$ of $w_{k m} w_{k s}$ as far to the left as possible by means of (37), and then change the onset $x_{k} x_{k+1} x_{k}$ according to (49). We get

$$
w_{k m} w_{k s} \equiv_{k+1} \alpha x_{k}^{2}\left(w_{k+1 m} w_{k+1 s}\right), \quad \alpha \neq 0 .
$$

For $k+1<s$ we apply the inductive supposition to the word in the parentheses and then by (49) and (37) transpose $x_{k}$ to its former position.

The case $k+1=s$, the basis of the induction on $k$, we prove by downward induction on $s$.

Let $k+1=s=n-1$. Then $m=n$. Let us first show that

$$
\underline{x_{n-1} x_{n}^{2} x_{n-1} x_{n}} x_{n} x_{n-1} \equiv_{n} \alpha x_{n-1} x_{n}^{2} x_{n-1}^{2} x_{n}^{2}+\beta x_{n-1} x_{n} x_{n-1}^{2} x_{n}^{3}, \quad \alpha \neq 0 .
$$


For this in the left hand side we transpose the first letter $x_{n}$ by means of (55) to the penultimate position, and then replace the ending $x_{n}^{3} x_{n-1}$ by (46). We get a linear combination of three words. One of them equals the second word of (58), while two other have the following forms.

$$
x_{n-1} x_{n} x_{n-1} x_{n} x_{n-1} x_{n}^{2}, x_{n-1} x_{n} x_{n-1} x_{n}^{2} x_{n-1} x_{n} .
$$

The former word by (36) transforms into the form (58). The latter one, after the application of (55) and the replacing of $x_{n-1} x_{n} x_{n-1}$ by (36), will have an additional term $x_{n-1} x_{n}^{3} x_{n-1}^{2} x_{n}$ to which it is possible to apply (48). The direct calculation of the coefficients shows that $\alpha=p_{n-1 n} p_{n n} \neq 0$.

Now let us multiply (58) by $x_{n-2}^{2}$ from the left and use (36) with $i=n-2$. We get that $w_{n-2 n} w_{n-2 n-1}$ with respect to $\equiv_{n-1}$ equals

$$
\gamma x_{n-2} x_{n-1} x_{n}^{2} x_{n-2} x_{n-1}^{2} x_{n}^{2}+\delta x_{n-2} x_{n-1} x_{n} x_{n-2} x_{n-1}^{2} x_{n}^{3}, \gamma \neq 0 .
$$

Let us apply (48) and then (49) and (48) to the second word. We get that this word with respect to $\equiv_{n-1}$ equals zero. The first word after application of (36) takes up the form

$$
\varepsilon w_{n-2 n-1} w_{n-2 n}+\varepsilon^{\prime} \underline{w n-2 n}_{n-1}^{2} x_{n-2} x_{n}^{2}, \quad \varepsilon \neq 0 .
$$

Thus, by Lemma 7.14, the basis of the induction on $s$ is proved.

Let us carry out the inductive step. Let $k+1=s<n-1$. If $m>s+1=$ $k+2$ then by the inductive supposition on $s$ we may write

$$
\begin{array}{r}
w_{k m} w_{k s}=\left(w_{k m} w_{k k+2}\right) x_{k+1} \equiv_{k+1} \alpha w_{k k+2} w_{k m} x_{k+1}= \\
\beta w_{k k+2} x_{k} x_{k+1} x_{k+2} x_{k+1} w_{k+3 m} .
\end{array}
$$

Taking into account (53) we may neglect the words starting with $x_{k+1}^{2}, x_{k+2}$ while transforming the underlined part:

$$
x_{k} x_{k+1} x_{k+2} x_{k+1} \equiv \gamma x_{k} x_{k+1}^{2} x_{k+2} \equiv \delta x_{k+1} x_{k} x_{k+1} x_{k+2} .
$$

In this way (60) is transformed into (56).

If $m=s+1=k+2<n$ then the relation (57) takes up the form

$$
w_{k m} w_{k s} \equiv_{k+1} \alpha x_{k}^{2}\left(w_{k+1 k+2} w_{k+1 k+3}\right) x_{k+2} x_{k+1} \text {. }
$$

Let us apply the inductive supposition with $k^{\prime}=k+1, s^{\prime}=k+2, m^{\prime}=k+3$ to the word in the parentheses. We get

$$
w_{k m} w_{k s} \equiv_{k+1} \alpha \varepsilon^{-1} x_{k}^{2} w_{k+1 k+3} w_{k+1 k+3} \underline{x_{k+2}^{2} x_{k+1}},
$$

or after an evident replacement

$$
w_{k m} w_{k s} \equiv_{k+1} \gamma x_{k}^{2} w_{k+1 k+3} w_{k+1 k+2} \cdot x_{k+1} x_{k+2}+\delta x_{k}^{2} w_{k+1 k+3}^{2} x_{k+1} x_{k+2}^{2} .
$$

In both terms we may transpose one letter $x_{k}$ to its former position by means of (49) and (37). We get

$$
w_{k m} w_{k s} \equiv_{k+1} \gamma^{\prime} \underline{w_{k k+3} w_{k k+1}} x_{k+2}+\delta^{\prime} w_{k k+3}^{2} x_{k+1} x_{k+2}^{2} \text {. }
$$


It is possible to apply (56) with $m^{\prime}=k+3, s^{\prime}=k+1$ to the first term since the case $m>s+1$ is completely considered. Therefore it is enough to show that the second term equals zero with respect to $\equiv_{k+1}$. When we transpose the third letter $x_{k+1}$ as far to the left as possible we get the word

$$
w_{k k+3} \underline{x_{k} x_{k+1} x_{k+2} x_{k+1}} w_{k+3 k+3} x_{k+2}^{2} .
$$

Taking into account (53) we may neglect the words starting with $x_{k+1}$ while transforming the underlined part:

$$
x_{k} \underline{x_{k+1} x_{k+2} x_{k+1}} \equiv x_{k+2} \underline{x_{k} x_{k+1}^{2}} \equiv x_{k+2} x_{k+1} x_{k} x_{k+1} .
$$

Therefore the word (63) equals $w_{k k+1} w_{k k+3} x_{k+2}^{2}$ with respect to $\equiv_{k+1}$ and it remains only to apply Lemma 7.14 twice.

Lemma 7.16. The set $B$ satisfies the conditions of Lemma 4.8.

Proof. By Lemmas 7.12 and 4.7 it is sufficient to show that in $U_{P}^{b}(\mathfrak{g})$ all words of the form $u_{0}, \ldots, u_{6}$ are linear combinations of lesser ones. The words $u_{0}$ are diminished by (37). The words $u_{1}, u_{2}$ have been presented in this way, without using $\left[x_{n-1} x_{n}^{2}\right]=0$, in the proof of the above theorem. The relation (53) shows that $u_{3} \equiv_{k+1} 0, u_{4} \equiv_{k+1} 0$. Lemma 7.15 with $s=m-1$ yields the necessary representation for $u_{5}$.

Let us prove by downward induction on $k$ that

$$
u_{6} \stackrel{d f}{=} u_{k n}^{2} x_{n} \equiv_{k+1} \varepsilon u_{k n} x_{n} u_{k n}, \quad \varepsilon \neq 0 .
$$

For $k=n-1$ this equality takes up the form (55). Let $k<n-1$. Let us transpose the second letter $x_{k}$ of $u_{k n}^{2} x_{n}$ as far to the left as possible by means of (37) and then apply (35). We get

$$
u_{k n}^{2} x_{n} \equiv_{k+1} \alpha x_{k}^{2}\left(u_{k+1 n}^{2} x_{n}\right), \quad \alpha \neq 0 .
$$

We may apply the inductive supposition to the term in the parentheses and then by $(35),(37)$ transpose one of $x_{k}$ 's to its former position.

Lemma 7.17. If $p_{11} \neq 1$ then the values of polynomials $[v]^{h}$, where $[v] \in B$, $v \neq x_{i} h \geq 1$ are not skew primitive, in particular, they are nonzero.

Proof. Note that for $n>2$ the sub-algebra generated by $x_{2}, \ldots x_{n}$ is defined by the Cartan matrix of the type $B_{n-1}$. This allows us to carry out the induction on $n$ with additional supposition that the statements 1 and 2 of Theorem $\mathrm{B}_{n}$ are valid for lesser values of $n$. It is convenient formally consider the one generated sub-algebras $\left\langle x_{i}\right\rangle$ as algebras of the type $B_{1}$. In this case for $n=1$ the lemma and the statements 1 and 2 are correct in the evident way. If $v$ starts with $x_{k} \neq x_{1}$ then we may directly use the inductive supposition. If $v=u_{1 m}$, one may literally repeat the arguments of Lemma 7.7 starting at the formula (41). Let $v=w_{1 m}$. If $m>2$ then by 
Lemma 7.8 we have $w_{1 m}=\left[x_{1}\left[w_{2 m}\right]\right]$. This provides a possibility to repeat the same arguments of Lemma 7.7 with $w$ in place of $u$.

Consider the last case $v=w_{12}$. By Lemma 7.8 we have

$$
\begin{aligned}
& {\left[w_{12}\right]=\left[w_{13}\right] x_{2}-p\left(w_{13}, x_{2}\right) x_{2}\left[w_{13}\right],} \\
& {\left[w_{13}\right]=x_{1}\left[w_{23}\right]-p\left(x_{1}, w_{23}\right)\left[w_{23}\right] x_{1} .}
\end{aligned}
$$

Applying the coproduct first to (66) then to (65) we may find the sum $\Sigma$ of all tensors $w^{(1)} \otimes w^{(2)}$ of $\Delta\left(\left[w_{12}\right]\right)$ with $\operatorname{deg}_{1}\left(w^{(2)}\right)=1, \operatorname{deg}_{k}\left(w^{(2)}\right)=0$, $k>1$ (in much the same way as (42)):

$$
\begin{aligned}
\Sigma & =\left(\varepsilon g_{1}\left[w_{23}\right] \otimes x_{1}\right)\left(x_{2} \otimes 1\right)-p\left(w_{13}, x_{2}\right)\left(x_{2} \otimes 1\right)\left(\varepsilon g_{1}\left[w_{23}\right] \otimes x_{1}\right) \\
& =\varepsilon g_{1}\left(\left[w_{23}\right] x_{2}-p\left(w_{13}, x_{2}\right) p\left(x_{2}, x_{1}\right) x_{2}\left[w_{23}\right]\right) \otimes x_{1} .
\end{aligned}
$$

For $n>2$, taking into account first the bicharacter property of $p$, then the equality $\left[x_{2}\left[w_{23}\right]\right]=x_{2}\left[w_{23}\right]-p\left(x_{2}, w_{23}\right)\left[w_{23}\right] x_{2}$, and next the following relations $p_{i j} p_{j i}=1, i-j>1 ; p_{11}^{-1}=p_{12} p_{21}=p_{22}^{-1}=p_{23} p_{32}$, we may write

$$
\Sigma=\varepsilon g_{1}\left(-p\left(w_{13}, x_{2}\right) p_{21}\left[x_{2} w_{23}\right]+\left(1-p_{11}^{-1}\right)\left[w_{23}\right] \cdot x_{2}\right) \otimes x_{1} .
$$

Consider the left hand side of this tensor on applying the inductive supposition. Note that $x_{2} w_{23}$ is a standard word and $\left[x_{2} w_{23}\right]$ equals $\left[x_{2}\left[w_{23}\right]\right]$. This super-letter is non-hard in $U_{P}(\mathfrak{g})$ since $x_{2} w_{23}$ contains the sub-word $x_{2}^{2} x_{3}$. Thus $\left[x_{2} w_{23}\right]$ is a linear combination of monotonous non-decreasing super-words in lesser super-letters. Among these super-words there is no $\left[w_{23}\right] \cdot x_{2}$ since $x_{2}>x_{2} w_{23}$. On the other hand, $\left[w_{23}\right] \cdot x_{2}$ is a monotonous non-decreasing super-word and hence its value in $U_{P}(\mathfrak{g})$ is a basis element. Therefore for $n>2$ the left hand side $W$ of $\Sigma$ is nonzero.

For $n=2$, by the definition $w_{23}=x_{2}, w_{13}=x_{1} x_{2}$, and the equality (67) takes up the form $\Sigma=\varepsilon g_{1}\left(1-p_{12} p_{22} p_{21}\right) x_{2}^{2} \otimes x_{1}$. Since $1 \neq p_{11}^{-1}=p_{12} p_{21}=$ $p_{22}^{-2}$, we get $\left(1-p_{12} p_{22} p_{21}\right)=1-p_{22}^{-1} \neq 0$. Therefore in this case $\Sigma \neq 0$ as well.

By [23, Corollary 10] and the inductive supposition the sub-algebra generated by $x_{2}, \ldots, x_{n}$ has no zero divisors. In particular $W^{h} \neq 0$ and $\Sigma^{h} \neq 0$ in any case.

It remains to note that for $n>1$ the sum of all tensors $w^{(1)} \otimes w^{(2)}$ of $\Delta\left(\left[w_{12}\right]^{h}\right)$ such that $\operatorname{deg}_{1}\left(w^{(2)}\right)=h, \operatorname{deg}_{k}\left(w^{(2)}\right)=0, k>1$ equals $\Sigma^{h}$, hence $\left[w_{12}\right]^{h}$ can not be skew-primitive.

Proof of Theorem $\mathrm{B}_{n}$. Since none of $u_{k m}, w_{k m}$ contains sub-words (30), Lemmas 7.16, 7.1, 4.8 imply the first statement.

If $[v] \in B$ is of finite height then by Lemma 7.13 and the homogeneous version of Definition 4.4 we have $[v]^{h}=0$. For $p_{11} \neq 1$ this contradicts Lemma 7.17. 
Along similar lines, by Lemma 4.9, every skew primitive homogeneous element has the form $[v]^{h}$. This, together with Lemma 7.17, proves the fourth statement and, for $p_{11} \neq 1$, the second one too.

If $p_{11}=1$ then by (47) we have $p_{n n}^{2}=1, p_{i i}=1, i<n$. Besides, $p_{i j} p_{j i}=1$ for all $i, j$. This means that the skew commutator is a quantum Lie operation. Hence all elements of $B$ are skew primitive. In the case $p_{n n}=1$ these elements span a color Lie algebra, while in the case $p_{n n}=-1$ they span a color Lie super-algebra. Now as in Theorem $\mathrm{A}_{n}$, we may use the $P B W$ theorem for the color Lie super-algebras.

The third statement will follow Theorem 5.2 and Lemmas 5.3, 7.12 if we prove that all super-letters (45) are zero in $U_{P}(\mathfrak{g})$. We have already proved that these super-letters are non-hard. Therefore it remains to use the homogeneous version of Definition 4.3 and Lemma 7.13.

Theorem $\mathbf{C}_{\boldsymbol{n}}$. Suppose that $\mathfrak{g}$ is of the type $C_{n}$, and $p_{i i} \neq-1,1 \leq i \leq n$, $p_{n-1 n-1}^{[3]} \neq 0$. Denote by $B$ the set of the following super-letters:

$$
\begin{array}{lll}
{\left[u_{k m}\right] \stackrel{d f}{=}\left[x_{k} x_{k+1} \ldots x_{m}\right],} & 1 \leq k \leq m \leq n ; \\
{\left[v_{k m}\right] \stackrel{d f}{=}\left[x_{k} x_{k+1} \ldots x_{n} \cdot x_{n-1} \ldots x_{m}\right],} & 1 \leq k<m<n ; \\
{\left[v_{k}\right] \stackrel{d f}{=}\left[u_{k n-1} u_{k n}\right],} & 1 \leq k<n .
\end{array}
$$

The statements given below are valid.

1. The values of the super-letters (69) in $U_{P}(\mathfrak{g})$ form the PBW-generators set.

2. Each of these super-letters has the infinite height in $U_{P}(\mathfrak{g})$.

3. The following relations with (23) form a Groebner-Shirshov system for $U_{P}(\mathfrak{g})$.

$$
\begin{aligned}
& {\left[u_{0}\right] \stackrel{d f}{=}\left[x_{k} x_{m}\right]=0, \quad 1 \leq k<m-1<n ;} \\
& {\left[u_{1}\right] \stackrel{d f}{=}\left[u_{k m} x_{k+1}\right]=0, \quad 1 \leq k<m \leq n,(k, m) \neq(n-2, n) \text {; }} \\
& {\left[u_{2}\right] \stackrel{d f}{=}\left[u_{k m} u_{k m+1}\right]=0, \quad 1 \leq k \leq m<n-1 ;} \\
& {\left[w_{3}\right] \stackrel{d f}{=}\left[v_{k m} x_{k+1}\right]=0, \quad 1 \leq k<m<n, k \neq m-2 ;} \\
& {\left[w_{4}\right] \stackrel{d f}{=}\left[v_{k k+1} x_{k+2}\right]=0, \quad 1 \leq k<n-1 \text {; }} \\
& {\left[w_{5}\right] \stackrel{d f}{=}\left[v_{k m} v_{k m-1}\right]=0, \quad 1 \leq k<m-1 \leq n-1 \text {; }} \\
& {\left[w_{6}\right] \stackrel{d f}{=}\left[u_{k n-1}^{3} x_{n}\right]=0, \quad 1 \leq k<n \text {. }}
\end{aligned}
$$

4. If $p_{11} \neq 1$ then the generators $x_{i}$ and their powers $x_{i}^{t}, x_{i}^{t l^{k}}$, such that $p_{i i}$ is a primitive $t$-th root of 1 together with the constants $1-g, g \in G$ form a basis of $\mathfrak{g}_{P}=L\left(U_{P}(\mathfrak{g})\right)$. Here $l$ is the characteristic of the ground field. 
5. If $p_{11}=1$ then the elements (69) and in the case of prime characteristic $l$ theirs $l^{k}$-th powers, together with the constants $1-g, g \in G$ form a basis of $\mathfrak{g}_{P}$.

In the case $C_{n}$ the algebra $U_{P}^{b}(\mathfrak{g})$ is defined by the same relations (35), (36), (37), where in (36) the last relation, $i=n-1$, is replaced with

$$
\begin{aligned}
x_{n-1}^{3} x_{n} & =p_{n-1 n} p_{n-1 n-1}^{[3]} x_{n-1}^{2} x_{n} x_{n-1}+ \\
& -p_{n-1 n}^{2} p_{n-1 n-1} p_{n-1 n-1}^{[3]} x_{n-1} x_{n} x_{n-1}^{2}+p_{n-1 n}^{3} p_{n-1 n-1}^{3} x_{n} x_{n-1}^{3} .
\end{aligned}
$$

By Corollary 2.5 we get the existence conditions

$$
\begin{gathered}
p_{i i}=p_{11}, \quad p_{i-1 i} p_{i i-1}=p_{11}^{-1}, 1<i<n, \\
p_{n-1 n} p_{n n-1}=p_{n n}^{-1}=p_{n-1 n-1}^{-2} ; p_{i j} p_{j i}=1, i-j>1 .
\end{gathered}
$$

Therefore the following relations are correct

$$
\begin{aligned}
& x_{i} x_{i+1}^{2} \equiv_{i+1} 0, \quad 1 \leq i<n ; \\
& x_{i} x_{i+1} x_{i} \equiv_{i+1} \alpha x_{i}^{2} x_{i+1}, \quad 1 \leq i<n-1, \alpha \neq 0 ; \\
& x_{n-1} x_{n} x_{n-1}^{2} \equiv_{n} \alpha x_{n-1}^{3} x_{n}+\beta x_{n-1}^{2} x_{n} x_{n-1}, \quad \alpha, \beta \neq 0 .
\end{aligned}
$$

The left multiplication by $x_{n-2}$ of the last relation implies

$$
x_{n-2} x_{n-1} x_{n} x_{n-1}^{2} \equiv_{n-1} 0 .
$$

Lemma 7.18. The brackets in $\left[v_{k m}\right],\left[v_{k}\right]$ are set according to the following recurrence formulae, where by the definition $v_{k n}=u_{k n}$.

$$
\begin{array}{ll}
{\left[v_{k m}\right]=\left[x_{k}\left[v_{k+1 m}\right]\right],} & \text { if } 1 \leq k<m-1<n-1 ; \\
{\left[v_{k k+1}\right]=\left[\left[v_{k k+2}\right] x_{k+1}\right],} & \text { if } 1 \leq k<n-1 ; \\
{\left[v_{k}\right]=\left[\left[u_{k n-1}\right]\left[u_{k n}\right]\right],} & \text { if } 1 \leq k<n .
\end{array}
$$

Proof. It is enough to use the properties $6 \mathrm{~s}, 1 \mathrm{~s}$ and $2 \mathrm{~s}$.

Lemma 7.19. If $[u],[v] \in B$ then one of the following statements is valid.

1) $[[u][v]]$ is not a standard nonassociative word;

2) uv contains a sub-word of one of the types $u_{0}, u_{1}, u_{2}, w_{3}, w_{4}, w_{5}, w_{6}$;

3) $[[u][v]] \in B$.

Proof. The first two formulae (77) coincide with (51) up to replacement of $v$ with $w$ provided $k+1 \neq n>m$. Obviously for $m<n$ the inequality $v_{k m}>$ $v_{r s}$ is equivalent to $w_{k m}>w_{r s}$, while $v_{k m}>u_{r s}$ is equivalent to $w_{k m}>w_{r s}$. Hence Lemmas 7.9, 7.10, 7.11 are still valid under the replacement of $w$ with $v$ :

$$
\begin{array}{ll}
{\left[\left[v_{k m}\right]\left[v_{r s}\right]\right]} & \text { is standard } \Leftrightarrow \quad s \geq m>k+1=r \vee(s<m \& r=k) ; \\
{\left[\left[u_{k m}\right]\left[v_{r s}\right]\right]} & \text { is standard } \Leftrightarrow k=r \vee k=m<r ; \\
{\left[\left[v_{k m}\right]\left[u_{r s}\right]\right]} & \text { is standard } \Leftrightarrow \quad r=k+1<m \vee r=k+1=m=s .
\end{array}
$$


Further, $v_{k}>v_{r}$ if and only if $k<r$, and under this condition $\left[\left[v_{k}\right]\left[v_{r}\right]\right]$ is not standard since $u_{k n}>u_{r n-1} u_{r n}$.

In a similar manner $v_{k}>u_{r m}$ is equivalent to $k<r$, while $v_{k}>v_{r m}$ is equivalent to $k \leq r$. Therefore none of the words $\left[\left[v_{k}\right]\left[u_{r m}\right]\right],\left[\left[v_{k}\right]\left[v_{r m}\right]\right]$ is standard since $u_{k n}>u_{r m}$ and $u_{k n}>v_{r m}$, respectively.

For the remaining two cases we have only two possibilities

$$
\begin{array}{ll}
{\left[\left[u_{k m}\right]\left[v_{r}\right]\right]} & \text { is standard } \Leftrightarrow r=k \leq m<n ; \\
{\left[\left[v_{k m}\right]\left[v_{r}\right]\right]} & \text { is standard } \Leftrightarrow r=k+1 \& k<m-1 .
\end{array}
$$

The treatment in turn of the eight possibilities (78), (79) proves the lemma.

Lemma 7.20. If a super-word $W$ equals one of the super-letters (70) or $[v]^{h},[v] \in B, h \geq 1$, then its constitution does not equal the constitution of any word in less then $W$ super-letters from $B$.

Proof. The proof is akin to Lemma 7.6 with the following tableaux:

$$
\begin{array}{ll}
{\left[u_{k m}\right]^{h},\left[u_{k m} x_{k+1}\right],\left[u_{k m} u_{k m+1}\right]} & \operatorname{deg}_{k}(u) \leq \operatorname{deg}_{m+1}(u) ; \\
{\left[v_{k m}\right]^{h},\left[v_{k m} x_{k+1}\right],\left[v_{k m} v_{k m-1}\right]} & 2 \operatorname{deg}_{k}(u) \leq \operatorname{deg}_{m-1}(u) ; \\
{\left[v_{k k+1} x_{k+2}\right]} & \operatorname{deg}_{k}(u)=0 ; \\
{\left[v_{k}\right]^{h}} & \operatorname{deg}_{k}(u) \leq \operatorname{deg}_{n}(u) ; \\
{\left[u_{k n-1}^{3} x_{n}\right]} & \operatorname{deg}_{k}(u) \leq 2 \operatorname{deg}_{n}(u) .
\end{array}
$$

Lemma 7.21. If $y=x_{i}, m-1 \neq i>k$ or $y=x_{i}^{2}, m-1=i>k$ then

$$
v_{k m} y \equiv_{k+1} 0 .
$$

Proof. For $i<m-1$, we may transpose $y$ by means of (37) to the left across $x_{n}^{2}$ and then use Lemma 7.3 with $m^{\prime}=n-1$.

If $y=x_{i}^{2}, m-1=i>k$ then by the above case, $i<m-1$, we get

$$
v_{k m} y=v_{k m+1} \underline{x_{m} x_{m-1}^{2}}=\underline{v_{k m+1} x_{m-1}}\left(\alpha x_{m} x_{m-1}+\beta x_{m-1} x_{m}\right) \equiv_{k+1} 0,
$$

where by definition $v_{k n}=u_{k n}$ and $u_{k n} x_{n-2} \equiv_{n-2} 0$, while $n-2=i>k$.

If $y=x_{i}, i=m>k$ then for $m=n-1$ we may use the inequality (76), while for $m<n-1$ we have $v_{k m} y=v_{k m+1} y_{1}$ where $y_{1}=x_{m}^{2}$. Hence we may use (82) replacing $m$ by $m+1$.

If $y=x_{i}, i>m>k$ then by (37) we get $v_{k m} y=\alpha v_{k i+1} x_{i} x_{i-1} x_{i} \cdot w$. Changing the underlined by (35), we may apply the previously considered cases: $m^{\prime}-1=i^{\prime}$, where $m^{\prime}=i+1, i^{\prime}=i$; and $i^{\prime}<m^{\prime}-1$, where $m^{\prime}=i+1$, $i^{\prime}=i-1$. 
If we multiply (71) by $x_{n}$ from the right and subtract (35) with $i=n-1$ multiplied from the left by $x_{n-1}^{2}$, then by means of $p_{n-1 n-1}^{-2}=p_{n n-1} p_{n-1 n}=$ $p_{n n}^{-1}$ we get

$$
x_{n-1}^{2} \underline{x_{n} x_{n-1} x_{n}} \equiv_{n} p_{n-1 n}\left(p_{n-1 n-1}^{[3]} x_{n-1} x_{n} x_{n-1}^{2} x_{n}-p_{n-1 n-1} x_{n-1}^{2} x_{n}^{2} x_{n-1}\right) .
$$

Let us first multiply this relation by $x_{n-2}^{2}$ from the left and then apply (35) to the underlined sub-word. Taking into account the relation $x_{n-2}^{2} x_{n-1}^{3} \equiv_{n-1} 0$, we get that the left hand side of the multiplied (83) equals $p_{n-1 n} p_{n n}(1+$ $\left.p_{n n}\right)^{-1} x_{n-2}^{2} x_{n-1}^{2} x_{n}^{2} x_{n-1}$ up to $\equiv_{n-1}$, i.e., it is proportional to the second term of the right hand side. As a result the relation below with $\alpha=p_{n-1 n-1}^{-1}(1+$ $\left.p_{n n}\right) \neq 0$ is correct.

$$
x_{n-2}^{2} x_{n-1}^{2} x_{n}^{2} x_{n-1} \equiv_{n-1} \alpha x_{n-2}^{2} x_{n-1} x_{n} x_{n-1}^{2} x_{n} .
$$

Lemma 7.22. If $k<s<m \leq n$ and as above $v_{k n}=u_{k n}$ then

$$
v_{k m} v_{k s} \equiv_{k+1} \varepsilon v_{k s} v_{k m}, \quad \varepsilon \neq 0 .
$$

Proof. Let us use downward induction on $k$. For this we first transpose the second letter $x_{k}$ of $v_{k m} v_{k s}$ as far to the left as possible by means of (37), and then change the onset $x_{k} x_{k+1} x_{k}$ according to (74). We get

$$
v_{k m} v_{k s} \equiv_{k+1} \alpha x_{k}^{2}\left(v_{k+1 m} v_{k+1 s}\right), \quad \alpha \neq 0 .
$$

For $k+1<s$ we may apply the inductive supposition to the word in the parentheses, and then transpose $x_{k}$ to its former position by (74), (37).

For $k+1=s$ we will use downward induction on $s$.

Let $k+1=s=n-1$. In this case $m=n$ and (86) becomes:

$$
v_{n-2 n} v_{n-2 n-1} \equiv_{n-1} \beta x_{n-2}^{2}\left(x_{n-1} \underline{x_{n} x_{n-1} x_{n}} x_{n-1}\right) .
$$

Let us replace the underlined part according to (35). Since $x_{n-2}^{2} x_{n-1} x_{n}^{2} \equiv_{n}$ 0 , we may continue by (84):

$$
\begin{aligned}
& \equiv_{n-1} \beta_{1} x_{n-2}^{2} x_{n-1}^{2} x_{n}^{2} x_{n-1} \equiv_{n-1} \beta_{2} \underline{x_{n-2}^{2} x_{n-1}} x_{n} x_{n-1}^{2} x_{n} \equiv_{n-1} \\
& \beta_{3} x_{n-2} x_{n-1} \underline{x_{n-2} x_{n}} x_{n-1}^{2} x_{n} \equiv_{n-1} \beta_{4} x_{n-2} x_{n-1} x_{n} \underline{x_{n-2} x_{n-1}^{2}} x_{n} .
\end{aligned}
$$

With the help of (35) we get

$$
=\varepsilon v_{n-2 n-1} v_{n-2 n}+\beta_{5} x_{n-2} \underline{x_{n-1} x_{n} x_{n-1}^{2}} x_{n-2} x_{n}, \quad \varepsilon \neq 0 .
$$

By (75) and (73) we see that the second term equals zero up to $\equiv_{n-1}$.

The inductive step on $s$ coincides the inductive step on $s$ in Lemma 7.15 up to replacing both the citations of Lemma 7.14 with the citations of Lemma 7.21 and $w$ with $v$.

Lemma 7.23. The set B satisfies the Lemma 4.8 conditions. 
Proof. According to Lemma 4.7 and Lemma 7.12 it is sufficient to show that words of the form $u_{0}, u_{1}, u_{2}, w_{3}, w_{4}, w_{5}, w_{6}$ are linear combinations of lesser words in $U_{P}(\mathfrak{g})$. The words $u_{0}$ are diminished by (37). The words $u_{1}, u_{2}$ have been diminished in Theorem $\mathrm{A}_{n}$ since in the case $C_{n}$ the words $u_{2}$ are independent of $x_{n}$, while $u_{1}$ depends on $x_{n}$ only if $u_{1}=x_{n-1} x_{n}^{2}$. The relation (81) shows that $w_{3} \equiv_{k+1} 0, w_{4} \equiv_{k+1} 0$. Lemma 7.22 with $s=m-1$ gives the required representation for $u_{5}$.

Consider the words $w_{6}$. For $k=n-1$ the relation (71) defines the required decomposition. Let $k<n-1$. Since $x_{1}, \ldots, x_{n-1}$ generate a sub-algebra of the type $A_{n-1}$, the decomposition of $u_{k n-2}^{3} x_{n-1}$ in the basis defined by Lemma 4.7 has the form

$$
u_{k n-2}^{3} x_{n-1}=\sum \alpha u_{m_{1} s_{1}} u_{m_{2} s_{2}} \cdots u_{m_{t} s_{t}},
$$

where $u_{m_{1} s_{1}} \leq u_{m_{2} s_{2}} \leq \ldots \leq u_{m_{t} s_{t}}$, that is $m_{1} \geq m_{2} \geq \ldots \geq m_{t}$, and $s_{i} \geq s_{i+1}$ if $m_{i}=m_{i+1}$. In particular, if $m_{1}=k$ then $m_{2}=\ldots=m_{t}=k$ and, due to the homogeneity, $t=3, s_{1}=n-1, s_{2}=s_{3}=n-2$. Therefore

$$
u_{k n-2}^{3} x_{n-1} \equiv_{k+1} \varepsilon u_{k n-1} u_{k n-2}^{2} \text {. }
$$

Along similar lines, the following relations are valid as well

$$
u_{k n-2}^{3} x_{n-1}^{2} \equiv_{k+1} \mu u_{k n-1}^{2} u_{k n-2}, \quad u_{k n-2}^{2} x_{n-1}^{3} \equiv_{k+1} 0 .
$$

Now let us multiply (35) with $i=n-2$ by $x_{n-1}$ from the right, and then add to the result the same relation multiplied by $p_{n-2 n-1}\left(1+p_{n-1 n-1}\right) x_{n-1}$ from the left. We get the following relation with $\alpha=p_{n-2 n-1}^{2} p_{n-1 n-1}^{[3]} \neq 0$,

$$
x_{n-2} x_{n-1}^{3}=\alpha x_{n-1}^{2} x_{n-2} x_{n-1}+\beta x_{n-1}^{3} x_{n-2} .
$$

Further, we may write

$$
u_{k n-1}^{3}=\beta_{1} u_{k n-2} u_{k n-3} \underline{x_{n-1} x_{n-2} x_{n-1}} u_{k n-1}, \quad \beta_{1} \neq 0,
$$

where for $k=n-2$ the term $u_{k n-3}$ is absent. Let us apply (35) with $i=n-2$ to the underlined word. Since $u_{k n-2} u_{k n-3} x_{n-1}^{2} \equiv_{n-1} 0$, we have got

$$
u_{k n-1}^{3} \equiv_{n-1} \beta_{2} u_{k n-2}^{2} u_{k n-3} \underline{x_{n-1}^{2} x_{n-2} x_{n-1}} .
$$

Let us apply (90). Taking into account the second of (89) we get

$$
u_{k n-1}^{3} \equiv_{k+1} \beta_{3} u_{k n-2}^{3} x_{n-1}^{3} \text {. }
$$

Let us multiply this relation from the right by $x_{n}$. By (71) we have

$$
u_{k n-1}^{3} x_{n} \equiv_{k+1} \alpha \underline{u_{k n-2}^{3} x_{n-1}} x_{n} x_{n-1}^{2}+\beta \underline{u_{k n-2}^{3} x_{n-1}^{2}} x_{n} x_{n-1} .
$$

By means of (88) and (89) we have got

$$
u_{k n-1}^{3} x_{n} \equiv_{k+1} \alpha_{1} u_{k n-1} x_{n} u_{k n-2}^{2} x_{n-1}^{2}+\beta_{1} u_{k n-1}^{2} x_{n} u_{k n-2} x_{n-1},
$$

and both of these words are less than $u_{k n-1}^{3} x_{n}$. 
Lemma 7.24. If $p_{11} \neq 1$ then the values of $[v]^{h}$, where $[v] \in B, v \neq x_{i}$, $h \geq 1$ are not skew primitive. In particular they are nonzero.

Proof. Note that for $n>3$ the algebra generated by $x_{2}, \ldots x_{n}$ is a subalgebra of the type $C_{n-1}$. Therefore we may use induction on $n$ with additional supposition that the theorem statements 1 and 2 are valid for the lesser values of $n$. We will formally consider the sub-algebra generated by $x_{n-1}, x_{n}$ as an algebra of the type $C_{2}$, and the sub-algebra generated by $x_{n}$ as an algebra of type $C_{1}$. In this case for $n=1$ the present lemma and the statements 1 and 2 are valid in obvious way.

If the first letter $x_{k}$ of $v$ is less than $x_{1}$ then we may use the inductive supposition directly. If $v=u_{1 m}$ then one may literally repeat arguments of Lemma 7.7 starting at (41).

If $v=v_{1 m}$ and $n>3$ then we may repeat arguments of Lemma 7.17 starting at (65) up to replacing $w$ with $v$. For $n=3$ in these arguments the formula (68) assumes the form

$$
\Sigma=\varepsilon g_{1}\left(-p\left(v_{13}, x_{2}\right) p_{21}\left[x_{2}^{2} x_{3}\right]+\left(1-p_{11}^{-1}\right)\left[x_{2} x_{3}\right] \cdot x_{2}\right) \otimes x_{1} .
$$

Therefore the left component of the tensor $\Sigma$ is a nonzero linear combination of the basis elements. For $n=2$ the set $B$ has no elements $v_{1 m}$ at all.

Consider the last case, $v=v_{1}=\left[u_{1 n-1}^{2} x_{n}\right]$. Let $S_{k}$ be the sum of all tensors of $\Delta\left(\left[u_{k n}\right]\right)=\sum u^{(1)} \otimes u^{(2)}$ with $\operatorname{deg}_{n}\left(w^{(1)}\right)=1, \operatorname{deg}_{k}\left(w^{(1)}\right)=0$, $k<n$. Evidently $S_{n}=x_{n} \otimes 1$. Let us show by downward induction on $k$ that $S_{k}=\left(1-p_{11}^{-1}\right) g\left(u_{k n-1}\right) x_{n} \otimes\left[u_{k n-1}\right]$ at $k<n$. We have

$$
\Delta\left(\left[u_{k n}\right]\right)=\Delta\left(x_{k}\right) \Delta\left(\left[u_{k+1 n}\right]\right)-p\left(x_{k}, u_{k+1 n}\right) \Delta\left(\left[u_{k+1 n}\right]\right) \Delta\left(x_{k}\right) .
$$

Consequently,

$$
S_{k}=\left(g_{k} \otimes x_{k}\right) S_{k+1}-p\left(x_{k}, u_{k+1 n}\right) S_{k+1}\left(g_{k} \otimes x_{k}\right) .
$$

This implies the required formula since by (72) at $k<n-1$ we have

$$
p\left(x_{k}, u_{k+1 n}\right) p\left(x_{n}, x_{k}\right)=p\left(x_{k}, u_{k+1 n-1}\right),
$$

while at $k=n-1$ we have $p\left(x_{n-1}, x_{n}\right) p\left(x_{n}, x_{n-1}\right)=p_{11}^{-1}$.

In a similar manner, consider the sum $S$ of all tensors of $\Delta\left(\left[u_{k n}^{2} x_{n}\right]\right)=$ $\sum w^{(1)} \otimes w^{(2)}$ with $\operatorname{deg}_{n}\left(w^{(1)}\right)=1, \operatorname{deg}_{i}\left(w^{(1)}\right)=0$, at $i<n$,

$$
\Delta\left(\left[\left[u_{1 n-1}\right]\left[u_{1 n}\right]\right]\right)=\Delta\left(\left[u_{1 n-1}\right]\right) \Delta\left(\left[u_{1 n}\right]\right)-p\left(u_{1 n-1}, u_{1 n}\right) \Delta\left(\left[u_{1 n}\right]\right) \Delta\left(\left[u_{1 n-1}\right]\right) .
$$

Since we know $S_{1}$, we may calculate $S$ :

$$
\begin{aligned}
S & =\left(g\left(u_{1 n-1}\right) \otimes\left[u_{1 n-1}\right]\right) S_{1}-p\left(u_{1 n-1}, u_{1 n}\right) S_{1}\left(g\left(u_{1 n-1}\right) \otimes\left[u_{1 n-1}\right]\right) \\
& =\left(1-p_{11}^{-1}\right) g\left(u_{1 n-1}^{2}\right) x_{n} \otimes\left(1-p\left(u_{1 n-1}, u_{1 n}\right) p\left(x_{n}, u_{1 n-1}\right)\right)\left[u_{1 n-1}\right]^{2} .
\end{aligned}
$$


By (72), using the bicharacter property of $p$, we have

$$
\begin{aligned}
& 1-p\left(u_{1 n-1}, u_{1 n}\right) p\left(x_{n}, u_{1 n-1}\right) \\
& =1-p\left(u_{1 n-1}, u_{1 n-1}\right) p_{n-1 n} p_{n n-1} \\
& =1-p_{n-1 n-1} p_{n-1 n-1}^{-2}=1-p_{11}^{-1} \neq 0 .
\end{aligned}
$$

Because of this, $S \neq 0$ and the sum of all tensors $w^{(1)} \otimes w^{(2)}$ with $\operatorname{deg}_{n}\left(w^{(1)}\right)=$ $h, \operatorname{deg}_{k}\left(w^{(1)}\right)=0, k<n$ of the basis decomposition of $\Delta\left(\left[v_{1}\right]^{h}\right)$ equals $S^{h} \neq 0$. Therefore $\left[v_{1}\right]^{h}$ is not skew primitive.

Proof of Theorem $\mathrm{C}_{n}$. For the first statement it will suffice to prove that all super-letters (69) are hard in $U_{P}(\mathfrak{g})$. Since none of $u_{k m}, v_{k m}$ contains a sub-word (30), Lemma 7.1 implies that $\left[u_{k m}\right],\left[v_{k m}\right]$ are hard.

If $\left[v_{k}\right]$ is not hard then, by the homogeneous version of Definition 4.3 , its value is a polynomial in lesser hard super-letters. In line with Lemmas 7.23 and 4.8, all hard super-letters belong to $B$. Therefore, by Lemma 7.20, $\left[v_{k}\right]=0$. Since $\operatorname{deg}_{n}\left(v_{k}\right)=1$ and $\operatorname{deg}_{n-1}\left(v_{k}\right)=2$, the equality $\left[v_{k}\right]=0$ is valid in the algebra $C^{\prime}$ which is defined by all relations of $U_{P}(\mathfrak{g})$, except ones of degree greater than 1 in $x_{n}$ and ones of degree greater than 2 in $x_{n-1}$, that is in the algebra defined by (35), (36) with $i<n-1$, and (37). These relations do not reverse the order of $x_{n-1}$ and $x_{n}$ in monomials since none of them has both $x_{n-1}$ and $x_{n}$. This implies that the sum of all monomials of $\left[v_{k}\right]=\left[u_{k n-1}\right] \cdot\left[u_{k n}\right]-p\left(u_{k n-1}, u_{k n}\right)\left[u_{k n}\right] \cdot\left[u_{k n-1}\right]$ in which $x_{n}$ is prefixed to $x_{n-1}$ equals zero in the above defined algebra $C^{\prime}$, that is $\left[u_{k n}\right] \cdot\left[u_{k n-1}\right]=0$. Especially, this equality is valid in $U_{P}(\mathfrak{g})$. Since, by Theorem 4.5, the superword $\left[u_{k n}\right] \cdot\left[u_{k n-1}\right]$ is a basis element, the first statement is proved.

If $[v] \in B$ is of finite height then, by Lemma 7.20 and the homogeneous version of Definition 4.4 , we have $[v]^{h}=0$. For $p_{11} \neq 1$ this contradicts Lemma 7.24. In a similar manner, according to Lemma 4.9, every skew primitive homogeneous element has the form $[v]^{h}$. This, together with Lemma 7.24 , proves the fourth statement and, for $p_{11} \neq 1$, the second one too. If $p_{11}=1$ then according to $(72)$ we have $p_{i i}=p_{i j} p_{j i}=1$ at all $i, j$. In particular, the skew commutator is a quantum Lie operation. Hence all elements of $B$ are skew primitive. These elements span a color Lie algebra. Now, as in Theorem $\mathrm{A}_{n}$, we may use the colored PBW theorem.

The third statement will follow from Theorem 5.2 and Lemmas 5.3, 7.19 provided we note that all super-letters $(70)$ are zero in $U_{P}(\mathfrak{g})$. We have proved already that these super-letters are non-hard. So it remains to use first the homogeneous version of Definition 4.3 and then Lemma 7.27. 
Theorem $\mathbf{D}_{n}$. Let $\mathfrak{g}$ be of the type $D_{n}$, and $p_{i i} \neq-1,1 \leq i \leq n$. Denote by $B$ the set of the following super-letters:

$$
\begin{array}{lll}
{\left[u_{k m}\right]} & \stackrel{d f}{=}\left[x_{k} x_{k+1} \ldots x_{m}\right], & 1 \leq k \leq m<n ; \\
{\left[e_{k m}\right]} & \stackrel{d f}{=}\left[x_{k} x_{k+1} \ldots x_{n-2} \cdot x_{n} x_{n-1} \ldots x_{m}\right], & 1 \leq k<m \leq n, \\
{\left[e_{n-1 n}\right] \stackrel{d f}{=} x_{n} .} &
\end{array}
$$

The statements given below are valid.

1. The values of $(100)$ in $U_{P}(\mathfrak{g})$ form the $P B W$-generators set.

2. Each of the super-letters (100) has infinite height in $U_{P}(\mathfrak{g})$.

3. The relations (23) together with the following ones form a GroebnerShirshov system for $U_{P}(\mathfrak{g})$.

$$
\begin{aligned}
& {\left[u_{0}\right] \stackrel{d f}{=}\left[x_{k} x_{m}\right]=0, \quad 1 \leq k<m-1<n,(k, m) \neq(n-2, n) ;} \\
& {\left[u_{1}\right] \stackrel{d f}{=}\left[u_{k m} x_{k+1}\right]=0, \quad 1 \leq k<m<n \text {; }} \\
& {\left[u_{1}^{\prime}\right] \stackrel{d f}{=}\left[x_{n-2} x_{n}^{2}\right]=0 \text {, }} \\
& {\left[u_{2}\right] \stackrel{d f}{=}\left[u_{k m} u_{k m+1}\right]=0, \quad 1 \leq k \leq m<n-1 \text {; }} \\
& {\left[v_{3}\right] \stackrel{d f}{=}\left[e_{k m} x_{k+1}\right]=0, \quad 1 \leq k<m \leq n, n-1 \neq k \neq m-2 ;} \\
& {\left[v_{4}\right] \stackrel{d f}{=}\left[e_{k k+1} x_{k+2}\right]=0, \quad 1 \leq k<n-2 ;} \\
& {\left[v_{4}^{\prime}\right] \stackrel{d f}{=}\left[e_{n-3 n-2} x_{n}\right]=0 \text {, }} \\
& {\left[v_{5}\right] \stackrel{d f}{=}\left[e_{k m} e_{k m-1}\right]=0, \quad 1 \leq k<m-1 \leq n-1 \text {; }} \\
& {\left[v_{6}\right] \stackrel{d f}{=}\left[u_{k m} e_{k n}\right]=0, \quad 1 \leq k \leq m<n, n-2 \leq m \text {. }}
\end{aligned}
$$

4. If $p_{11} \neq 1$, then the generators $x_{i}$, their powers $x_{i}^{t}, x_{i}^{t l^{k}}$, such that $p_{i i}$ is a primitive $t$-th root of 1 , together with the constants $1-g, g \in G$ form a basis of $\mathfrak{g}_{P}=L\left(U_{P}(\mathfrak{g})\right)$. Here $l=\operatorname{char}(\mathbf{k})$.

5. If $p_{11}=1$, then the elements of $B$ and, for $l>0$, their $l^{k}$-th powers together with the constants $1-g, g \in G$ form a basis of $\mathfrak{g}_{P}$.

In the case $D_{n}$ the algebra $U_{P}^{b}(\mathfrak{g})$ can be defined by the condition that the sub-algebras $U_{n-1}$ and $U_{n}$ generated, respectively, by $x_{1}, \ldots, x_{n-1}$ and $x_{1}, \ldots, x_{n-2}, x_{n-1}^{\prime}=x_{n}$ are quantum universal enveloping algebras of the type $A_{n-1}$, and by the only additional relation

$$
\left[x_{n-1} x_{n}\right]=0 .
$$

The existence conditions take up the form

$$
\begin{aligned}
& p_{i i}=p_{n n}=p_{11}, p_{i+1 i} p_{i i+1}=p_{n-2 n} p_{n n-2}=p_{11}^{-1}, \quad \text { if } 1 \leq i<n, \\
& p_{n-1 n} p_{n n-1}=p_{i j} p_{j i}=1, \quad \text { if } i-j>1 \&(i, j) \neq(n, n-2) .
\end{aligned}
$$


Lemma 7.25. The brackets in (100) are set up by the recurrence formulae

$$
\begin{aligned}
& {\left[e_{k m}\right]=\left[x_{k}\left[e_{k+1 m}\right]\right], \quad \text { if } 1 \leq k<m-1<n, k \neq n-1 ;} \\
& {\left[e_{k k+1}\right]=\left[\left[e_{k k+2}\right] x_{k+1}\right], \quad \text { if } 1 \leq k<n-1 .}
\end{aligned}
$$

Proof. It is enough to use the properties $6 \mathrm{~s}, 1 \mathrm{~s}$, and $2 \mathrm{~s}$.

Lemma 7.26. If $[u],[v] \in B$, then one of the statements below is correct.

1) $[[u][v]]$ is not a standard nonassociative word;

2) uv contains a sub-word of one of the types $u_{0}, u_{1}, u_{1}^{\prime}, u_{2}, v_{3}, v_{4}, v_{4}^{\prime}, v_{5}, v_{6}$;

3) $[[u][v]] \in B$.

Proof. The formulae (104) coincides with (51) at $k \neq n-1$ up to replacing $e$ by $w$. The inequality $e_{k m}>e_{r s}$ is set up by the same conditions, $k<$ $r \vee(k=r \& m<s)$, as the inequality $w_{k m}>w_{r s}$ does. Likewise $u_{k m}>e_{r s}$ is set up by the same condition, $k \leq r$, as $u_{k m}>w_{r s}$ does. Therefore Lemmas $7.9,7.10,7.11$ remain valid with $e$ in place of $w$ :

$$
\begin{array}{ll}
{\left[\left[e_{k m}\right]\left[e_{r s}\right]\right]} & \text { is standard } \Leftrightarrow s \geq m>k+1=r \vee(s<m \& r=k) ; \\
{\left[\left[u_{k m}\right]\left[e_{r s}\right]\right]} & \text { is standard } \Leftrightarrow k=r \vee k=m<r ; \\
{\left[\left[e_{k m}\right]\left[u_{r s}\right]\right]} & \text { is standard } \Leftrightarrow r=k+1<m \vee r=k+1=m=s .
\end{array}
$$

By looking over all of these possibilities we get the lemma statement.

Lemma 7.27. If a super-word $W$ equals one of the super-letters (101) or $[v]^{h},[v] \in B, h \geq 1$ then its constitution does not equal the constitution of any super-word in less than $W$ super-letters from $B$.

Proof. The proof is similar to the one of Lemma 7.6 with the tableaux

$$
\begin{aligned}
& {\left[u_{k m}\right]^{h}, \quad\left[u_{k m} x_{k+1}\right], \quad\left[u_{k m} u_{k m+1}\right] \quad \operatorname{deg}_{k}(u) \leq \operatorname{deg}_{m+1}(u) ;} \\
& {\left[e_{k m}\right]^{h}, \quad\left[e_{k m} x_{k+1}\right], \quad\left[e_{k m} e_{k m-1}\right], \quad m<n \quad 2 \operatorname{deg}_{k}(u) \leq \operatorname{deg}_{m-1}(u) \text {; }} \\
& {\left[e_{k n}\right]^{h}, \quad\left[e_{k n} x_{k+1}\right], \quad\left[e_{k n} e_{k n-1}\right] \quad \operatorname{deg}_{k}(u) \leq \operatorname{deg}_{m-1}(u) ;} \\
& {\left[e_{k k+1} x_{k+2}\right] \quad \operatorname{deg}_{k}(u)=0 ;} \\
& \text { (106) } \begin{array}{ll}
{\left[e_{n-3 n-2} x_{n}\right]} & \operatorname{deg}_{n-3}(u)=0 ; \\
{\left[u_{k n-2} e_{k n}\right]} & \operatorname{deg}_{k}(u) \leq \operatorname{deg}_{n-1}(u)+\operatorname{deg}_{n}(u) ;
\end{array} \\
& {\left[u_{k n-1} e_{k n}\right] \quad \operatorname{deg}_{k}(u) \leq \operatorname{deg}_{n}(u) .}
\end{aligned}
$$

Lemma 7.28. If $y=x_{i}, \quad m-1 \neq i>k$ or $y=x_{i}^{2}, \quad m-1=i>k$ then

$$
e_{k m} y \equiv_{k+1} 0 \text {. }
$$

Proof. If $i<m-1, m \neq n$, or $m=n, i<n-2$, then with the help of (37) and (102) it is possible to permute $y$ to the left beyond $x_{n}$ and then to use Lemma 7.3 for $U_{n-1}$.

If $m=n, i=n-2$ then we may use Lemma 7.3 for $U_{n}$. 
If $y=x_{i}^{2}, m-1=i>k$ then for $m<n$ by the above case we get

$$
e_{k m} y=e_{k m+1} x_{m} x_{m-1}^{2}=\underline{e_{k m+1} x_{m-1}}\left(\alpha x_{m} x_{m-1}+\beta x_{m-1} x_{m}\right) \equiv_{k+1} 0 .
$$

For $m=n$ we have $e_{k n} x_{n-1}^{2}=\alpha u_{k n-2} x_{n-1}^{2} x_{n} \equiv_{n-1} 0$ since the underlined part belongs to $U_{n-1}$.

If $y=x_{i}, i=m>k$ then for $m=n$ we may use Lemma 7.3 applied to $U_{n}$; for $m=n-1$ we may use the same lemma applied to $U_{n-1}$ provided that beforehand we permute $x_{n}$ with $y$ by (102); for $m<n-1$ we may first rewrite $e_{k m} y=e_{k m+1} y_{1}$, where $y_{1}=x_{m}^{2}$, and then use (108) with $m+1$ in place of $m$.

If $y=x_{i}, i>m>k$ then for $i<n$ we have $e_{k m} y=\alpha e_{k i+1} x_{i} x_{i-1} x_{i} \cdot v$. Replacing the underlined word by (35) in $U_{n-1}$, we may use the previously considered cases: $m^{\prime}-1=i^{\prime}$, where $m^{\prime}=i+1, i^{\prime}=i$; and $i^{\prime}<m^{\prime}-1$, where $m^{\prime}=i+1, i^{\prime}=i-1$. For $i=n$, and $m=n-1$ we have $e_{k n-1} x_{n}=$ $\alpha u_{k n-2} x_{n}^{2} x_{n-1}$ and one may apply Lemma 7.3 to $U_{n}$. Finally, for $i=n$ and $m<n-1$ we get

$$
\begin{aligned}
e_{k m} x_{n}= & \beta_{1} u_{k n-2} x_{n} x_{n-1} x_{n-2} x_{n} \cdot v=\beta_{2} u_{k n-2} x_{n-1} \underline{x_{n} x_{n-1} x_{n}} \cdot v= \\
& \beta_{3} \underline{u_{k n-2} x_{n-1} x_{n-2}} x_{n}^{2} \cdot v+\beta_{4} u_{k n-2} \underline{x_{n-1} x_{n}^{2}} x_{n-2} \cdot v .
\end{aligned}
$$

One may apply first Lemma 7.3 for $U_{n-1}$ to the underlined sub-word of the first term, and then, after (102), Lemma 7.3 for $U_{n}$ to the second term.

Lemma 7.29. If $k<s<m \leq n$ then $e_{k m} e_{k s} \equiv_{k+1} \varepsilon e_{k s} e_{k m}, \varepsilon \neq 0$.

Proof. Let us carry out downward induction on $k$. The largest value of $k$ equals $n-2$. In this case $s=n-1, m=n$ and we have

$$
\begin{gathered}
\frac{x_{n-2} x_{n} \cdot x_{n-2}}{\beta x_{n-2} x_{n-1}} x_{n-2} x_{n-1} \equiv_{n}^{2} \equiv_{n-2}^{2} \underline{x_{n}^{2} x_{n-1}}=\alpha \underline{x_{n-2}^{2} x_{n-1}} x_{n-1}^{2} x_{n} \cdot x_{n-1} \equiv_{n-2} x_{n-1} .
\end{gathered}
$$

Let us first transpose the second letter $x_{k}$ of $e_{k m} e_{k s}$ as far to the left as possible by (37), and then replace the onset $x_{k} x_{k+1} x_{k}$ by (38). We get

$$
e_{k m} e_{k s} \equiv_{k+1} \alpha x_{k}^{2}\left(e_{k+1 m} e_{k+1 s}\right), \quad \alpha \neq 0 .
$$

For $k+1<s$ it suffices to apply the inductive supposition to the word in the parentheses and then by (38) and (37) to put $x_{k}$ to the proper place.

For $k+1=s$ one may use downward induction on $s$. The basis of this induction, $s=n-1$, has been proved, see (109). For $k<n-3$ the inductive step on $s$ coincides with the one of Lemma 7.15 with $e$ in place of $w$ since in this case the active variables $x_{k}, x_{k+1} q$-commute with $x_{n}$. If $k=n-3$ then in consideration of Lemma 7.15 the variable $x_{k+1}=x_{n-2}$ is transposed across $x_{n}$ twice: In (60) and in the second word of (62). 
In (60) with $k=n-3$ we have $s=n-2, m=n$; and (60) becomes

$$
e_{n-3 n} e_{n-3 n-2} \equiv_{n-2} \beta e_{n-3 n-1} x_{n-3} x_{n-2} x_{n} x_{n-2} .
$$

In view of Lemma 7.28 , we may transform the underlined part in $U_{n}$ neglecting the words starting with $x_{n-2}^{2}$ and $x_{n}$ in much the same way as in (61), with $x_{n}$ in place of $x_{k+1}$. So (111) reduces to the required form.

The second word of (62) with $k=n-3$ assumes the form $e_{n-3 n}^{2} x_{n-2} x_{n-1}^{2}=$ $e_{n-3 n} x_{n-3} x_{n-2} x_{n} x_{n-2} x_{n-1}^{2}$. By Lemma 7.3 applied to $U_{n}$, the underlined word is a linear combination of words starting with $x_{n-2}$ and $x_{n}$. However, by Lemma 7.28 both $e_{n-3 n} x_{n-2}$ and $e_{n-3 n} x_{n}$ equal zero up to $\equiv_{n-2}$.

Lemma 7.30. The set $B$ satisfies the conditions of Lemma 4.8.

Proof. By Lemmas 7.26 and 4.7 one need show only that in $U_{P}^{b}(\mathfrak{g})$ the words (101) are linear combinations of lesser ones. The words $v_{6}$ with $m=n-2$, and $u_{0}, u_{1}, u_{1}^{\prime}, u_{2}$ have the required decomposition since they belong either to $U_{n-1}$ or to $U_{n}$. Lemma 7.28 shows that $v_{3} \equiv_{k+1} 0, v_{4} \equiv_{k+1} 0, v_{4}^{\prime} \equiv_{k+1}$ 0 . Lemma 7.29 with $s=m-1$ yields the required representation for $v_{5}$. Consider $v_{6}$ with $m=n-1$. Let us prove by downward induction on $k$ that

$$
u_{k n-1} e_{k n} \equiv_{k+1} \varepsilon e_{k n} u_{k n-1}, \quad \varepsilon \neq 0 .
$$

For $k=n-1$ this equality assumes the form (102). Let $k<n-1$. Let us transpose the second letter $x_{k}$ of $u_{k n-1} e_{k n}$ as far to the left as possible in $U_{n-1}$. After an application of (35) we get

$$
u_{k n-1} e_{k n} \equiv_{k+1} \alpha x_{k}^{2}\left(u_{k+1 n-1} e_{k+1 n}\right), \quad \alpha \neq 0 .
$$

It suffices to apply the inductive supposition to the term in the parentheses, and then by (35) and (37) for $U_{n}$ to move $x_{k}$ to the proper place.

Lemma 7.31. If $p_{11} \neq 1$ then the values of $[v]^{h}$, where $[v] \in B, v \neq x_{i}$, $h \geq 1$ are not skew primitive, in particular they are nonzero.

Proof. One need consider only super-letters that belong neither to $U_{n-1}$ nor to $U_{n}$. That is $\left[e_{k m}\right]$ with $m<n$. We use induction on $n$.

For $n=3$ the algebra of the type $D_{3}$ reduces to the algebra of the type $A_{3}$ with a new ordering of variables $x_{2}>x_{1}>x_{3}$. Therefore we may use Theorem $\mathrm{A}_{n}$, after the decomposition below of $e_{12}$ in the PBW-basis:

$$
\left[\left[x_{1} x_{3}\right] x_{2}\right]=-p_{12} p_{32}\left[x_{2}\left[x_{1} x_{3}\right]\right]+\beta\left[x_{1} x_{3}\right] \cdot x_{2} \text {. }
$$

Let $n>3$. If $k>1$ then the inductive supposition works. For $k=1$, $m>2$ we have $e_{1 m}=\left[x_{1}\left[e_{2 m}\right]\right]$, and one may repeat the arguments of Lemma 7.7 with $e$ in place of $u$ starting at (41). If $m=2$ then we may repeat the arguments of Lemma 7.17 with $e$ on place of $w$ starting at (65).

Proof of Theorem $\mathrm{D}_{n}$. For the first statement it will suffice to prove that all super-letters $(100)$ are hard in $U_{P}^{b}(\mathfrak{g})$.

Since none of $u_{k m}$ contains sub-words (30), [u $\left.u_{k m}\right]$ are hard. 
Suppose $\left[e_{k m}\right]$ is non-hard. By Lemmas 7.30 and 4.8 all hard super-letters belong to $B$. Thus, by Lemma 7.27 , we get $\left[e_{k m}\right]=0$. Since $\operatorname{deg}_{n}\left(e_{k m}\right)=$ $\operatorname{deg}_{n-1}\left(e_{k m}\right)=1$, the equality $\left[e_{k m}\right]=0$ is also valid in the algebra $D^{\prime}$ defined by the same relations as $U_{P}^{b}(\mathfrak{g})$ is, except $\left[x_{n-2} x_{n}^{2}\right]=0$ and $\left[x_{n-2} x_{n-1}^{2}\right]$ $=0$. Let us equate to zero all monomials in all the defining relations of $D^{\prime}$, except $\left[x_{n-1} x_{n}\right]$. Consider the algebra $R^{\prime}$ defined by (102) and by the resulting system of monomial relations. It is easy to verify that the mentioned relations system $\Sigma$ of $R^{\prime}$ is closed under the compositions. Since $e_{k m}$ contains none of leading words of $\Sigma$, the super-letter $\left[e_{k m}\right]$ is nonzero in $R^{\prime}$, and so in $D^{\prime}$ too. This contradiction proves the first statement.

If $[v] \in B$ is of finite height then by Lemma 7.27 and the homogeneous version of Definition 4.4 we have $[v]^{h}=0$. For $p_{11} \neq 1$ this contradicts Lemma 7.31. In a similar manner, by Lemma 4.9, every skew primitive homogeneous element has the form $[v]^{h}$. This, together with Lemma 7.31, proves both the fourth statement and the second one with $p_{11} \neq 1$.

If $p_{11}=1$ then by (103) we have $p_{i i}=p_{i j} p_{j i}=1$ for all $i, j$. This means that the skew commutator itself is a quantum Lie operation. Hence all elements of $B$ are skew-primitive. These elements span a color Lie superalgebra. Now, as in Theorem $\mathrm{A}_{n}$, one may use the PBW theorem for color Lie super-algebras.

For the third statement it will suffice to show that all super-letters (101) are zero in $U_{P}(\mathfrak{g})$. We have proved already that they are non-hard. Therefore it remains to use the homogeneous version of Definition 4.3 and Lemma 7.27.

\section{Conclusion.}

We see that in all Theorems $\mathrm{A}_{n}-\mathrm{D}_{n}$ the lists of hard super-letters are independent of the parameters $p_{i j}$. Therefore if we put $p_{i j}=1$, we get a basis of the ground Lie algebra $\mathfrak{g}$. It is easy to see that this basis coincides with the basis defined by Lalonde and Ram in [28, Figure 1]. This fact signifies that the Lalonde-Ram basis of the ground Lie algebra with the skew commutator in place of the Lie operation coincides with the set of all hard super-letters of an arbitrary quantification. It is very interesting to clarify how general this statement is. On the one hand, this does not hold without exception for all quantum enveloping algebras since in Theorems $\mathrm{A}_{n}-\mathrm{D}_{n}$ a restriction does exist. If $p_{i i}=-1,1 \leq i<n, n>2$ then it is easy to see by means of Diamond Lemma that the sets of hard super-letters are infinite, while the ground Lie algebra is of finite dimension. On the other hand, this is not a specific property of Lie algebras defined by the Serre relations. By the Shirshov theorem [40] any Lie polynomial can be reduced to a linear combination of standard nonassociative words. 
Corollary 8.1. If $\mathfrak{g}$ is defined by the only relation $f=0$, where $f$ is a linear combination of standard nonassociative words, then the set of all hard in $U_{P}(\mathfrak{g})$ super-letters coincides with the Hall-Shirshov basis of $\mathfrak{g}$ with the skew commutator in place of the Lie operation.

Proof. The only relation $f^{*}=0$ forms a Groebner-Shirshov system since, according to 1s, none of onsets of its leading word, say $w$, coincides with a proper terminal of $w$. Consequently, a super-letter $[u]$ is hard if and only if $u$ does not contain $w$ as a sub-word. We see that this criteria is independent of $p_{i j}$ as well.

Furthermore, the third statement of Theorem $\mathrm{A}_{n}$ shows that $U_{P}^{b}(\mathfrak{g})$ can be defined by the following relations in the PBW-generators $X_{u}=[u]$.

$$
\begin{aligned}
& {\left[X_{u}, X_{v}\right]=0, \quad u>v, \quad[[u][v]] \notin B} \\
& {\left[X_{u}, X_{v}\right]=X_{u v}, \quad[[u][v]] \in B \text {. }}
\end{aligned}
$$

This is an argument in favor of considering the super-letters PBW-generators $\mathbf{k}[G]$-module as a quantum analogue of a Lie algebra. However in the cases $B_{n}, C_{n}, D_{n}$ the defining relations in the PBW-generators became more complicated. For example,

$$
\begin{array}{lll}
B_{n}: & {\left[\left[u_{k n-1}\right]\left[w_{k n}\right]\right]=\alpha\left[u_{k n}\right]^{2},} & \alpha \neq 0 \text { if } p_{n n} \neq 1 \\
C_{n}: & {\left[\left[u_{k n-2}\right]\left[v_{k n-1}\right]\right]=\alpha\left[v_{k}\right]+\beta\left[u_{k n}\right] \cdot\left[u_{k n-1}\right],} & \beta \neq 0 \text { if } p_{11} \neq 1 \\
D_{n}: & {\left[\left[u_{k n-2}\right]\left[e_{k n-1}\right]\right]=\alpha\left[e_{k n}\right] \cdot\left[u_{k n-1}\right],} & \alpha \neq 0 \text { if } p_{11} \neq \pm 1 .
\end{array}
$$

Also it is interesting that for $p_{11} \neq 1$ the algebra $\mathfrak{g}_{P}$ turns out to be very simple in structure. Only unary quantum Lie operations can be nonzero. Other ones may be defined, but due to the homogeneity their values equal zero. In particular, if $p_{11}^{t} \neq 1$ then without exception all quantum Lie operations have zero values. This provides reason enough to consider $U_{P}(\mathfrak{g})=$ $U\left(\mathfrak{g}_{P}\right)$ as an algebra of 'commutative' quantum polynomials or quantum 'symmetric' algebra. This statement is still retained for a large class of the quantum universal enveloping algebras of homogeneous components of other Kac-Moody algebras defined by the Gabber-Kac relations (11) (see M. Rosso [38, Theorem 15, and Remark 1] $)^{1}$. One may note that if a semigroup generated by $p_{i j} p_{j i}$ does not contain 1 , then $G\left\langle x_{1}, \ldots, x_{n}\right\rangle$ itself is a 'commutative' quantum polynomial algebra merely since in this case there exists no nonzero quantum Lie operation at all. In another extreme case when $p_{i j} p_{j i}=1$ for all $i, j$, the 'commutative' quantum variables commute by $x_{i} x_{j}=p_{i j} x_{j} x_{i}$ (see [38, Example 1, p. 409]).

\footnotetext{
${ }^{1}$ We note, howerever, that Proposition 17 and Corollary 18 of [38] are wrong: The quantum shuffles may have finite heights.
} 
Acknowledgments. The author is grateful to J.A. Montaraz, the director of the FES-C UNAM, S. Rodríguez-Romo, and A.V. Lara Sagahon for providing facilities for the research and also to L.A. Bokut', R. Bautista, and N. Andruskiewitsch for helpful comments on the subject matter.

\section{References}

[1] N. Andruskiewitsch and H.J. Schneider, Lifting of quantum linear spaces and pointed Hopf algebras of order $p^{3}$, J. Algebra, 209 (1998), 658-691, MR 99k:16075, Zbl 0919.16027.

[2] C. Bautista, A Poincarè-Birkhoff-Witt theorem for generalized Lie color algebras, J. Math. Phys., 39(7) (1998), 3829-3843, MR 99i:17013, Zbl 0928.17009.

[3] K.I. Beidar, W.S. Martindale III and A.V. Mikhalev, Rings with Generalized Identities, Pure and Applied Mathematics, 196, Marcel Dekker, New York-Basel-Hong Kong, 1996, MR 97g:16035, Zbl 0847.16001.

[4] G.M. Bergman, The diamond lemma for ring theory, Adv. Math., 29(2) (1978), 178218, MR 81b:16001, Zbl 0377.16013.

[5] L.A. Bokut', Unsolvability of the word problem and subalgebras of finitely presented Lie algebras, Izv. Akad. Nauk. Ser. Mat., 36(6) (1972), 1173-1219, MR 48 \#8588.

[6] _ Imbeddings into simple associative algebras, Algebra Logic, 15(2) (1976), 117-142, MR 58 \#22167.

[7] L.A. Bokut' and A.A. Klein, Serre relations and Groebner-Shirshov bases for simple Lie algebras I, II, Internat. J. Algebra Comput., 6(4) (1996), 389-412, MR 97k:17005, Zbl 0866.17007, Zbl 0866.17008.

[8] L.A. Bokut' and G.P. Kukin, Algorithmic and Combinatorial Algebra, Mathematics and Its Applications, 255, Kluwer Academic Publishers, Dordrecht-Boston-London, 1994, MR 95i:17002, Zbl 0826.17002.

[9] L.A. Bokut' and P. Malcolmson, Groebner bases for quantum enveloping algebras, Israel J. Math., 96 (1996), 97-113, MR 97k:17013, Zbl 0910.17004.

[10] R. Borcherds, Generalized Kac-Moody algebras, J. Algebra, 11 (1988), 501-512, MR 89g:17004, Zbl 0644.17010.

[11] K.T. Chen, R.H. Fox and R.C. Lyndon, Free differential calculus IV, the quotient groups of the lower central series, Ann. Math., 68 (1958), 81-95, MR 21 \#1330, Zbl 0142.22304.

[12] G. Clift, Crystal bases and Young tableaux, J. Algebra, 202(1) (1998), 10-35.

[13] P.M. Cohn, Sur le critère de Friedrichs pour les commutateur dans une algèbre associative libre, C. R. Acad. Sci. Paris, 239(13) (1954), 743-745, MR 16,562b, Zbl 0059.03101.

[14] _ Universal Algebra, Harper and Row, New-York, 1965, MR 31 \#224, Zbl 0141.01002.

[15] V.G. Drinfeld, Hopf algebras and the Yang-Baxter equation, Soviet Math. Dokl., 32 (1985), 254-258, MR 87h:58080, Zbl 0588.17015.

[16] K.O. Friedrichs, Mathematical aspects of the quantum theory of fields, V, Commun. Pure Appl. Math., 6 (1953), 1-72, MR 15,80b, Zbl 0052.44504. 
[17] O. Gabber and V. Kac, On defining relations of certain infinite-dimensional Lie algebras, Bull. Am. Math. Soc., New Ser., 5(2) (1981), 185-189, MR 84b:17011, Zbl 0474.17007.

[18] M. Jimbo, A q-difference analogue of $U(\mathfrak{g})$ and the Yang-Baxter equation, Lett. Math. Phys., 10 (1985), 63-69, MR 86k:17008, Zbl 0587.17004.

[19] S.-J. Kang, Quantum deformations of generalized Kac-Moody algebras and their modules, J. Algebra, 175 (1995), 1041-1066, MR 96k:17023, Zbl 0835.17016.

[20] M. Kashiwara, Crystallizing the q-analogue of universal enveloping algebras, Commun. Math. Phys., 133 (1990), 249-260, MR 92b:17018, Zbl 0724.17009.

[21] _ On crystal bases of the q-analog of universal enveloping algebras, Duke Math. J., 63(2) (1991), 465-516, MR 93b:17045, Zbl 0739.17005.

[22] V.K. Kharchenko, An algebra of skew primitive elements, Algebra Logic, 37(2) (1998), 181-224; English translation 101-126; QA/0006077, Zbl 0917.16017.

[23] A quantum analogue of the Poincarè-Birkhoff-Witt theorem, Algebra Logic, 38(4) (1999), 476-507; English translation 259-276; QA/0005101, MR 2001f:16075.

[24] _ An existence condition for multilinear quantum operations, J. Algebra, 217 (1999), 188-228, MR 2001d:16059, Zbl 0943.17016.

[25] _ Character Hopf algebras and quantizations of Lie algebras, Dokl. Math., 60(3) (1999), 328-329.

[26] Skew primitive elements in Hopf algebras and related identities, J. Algebra, 238(2) (2001), 534-559, CMP 1823773.

[27] A. Kuniba, K.C. Misra, M. Okado, T. Takagi and J. Uchiyama, Crystals for Demazure modules of classical affine Lie algebras, J. Algebra, 208 (1998), 185-215, MR 99h:17008.

[28] M. Lalonde and A. Ram, Standard Lyndon bases of Lie algebras and enveloping algebras, Trans. Am. Math. Soc., 347(5) (1995), 1821-1830, MR 95h:17013, Zbl 0833.17003.

[29] M. Lothaire, Combinatorics on words, Encyclopedia of Mathematics and its Applications, 17, Addison-Wesley Publ. Co., 1983, MR 84g:05002, Zbl 0514.20045.

[30] G. Lusztig, Quantum groups at roots of 1, Geom. Dedicata, 35(1-3) (1990), 89-113, MR 91j:17018, Zbl 0714.17013.

[31] Introduction to Quantum Groups, Progress in Mathematics, 10, Birkhauser Boston, 1993, MR 94m:17016, Zbl 0788.17010.

[32] R.C. Lyndon, A theorem of Friedrichs, Michigan Math. J., 3(1) (1955-1956), 27-29, MR 18,659c, Zbl 0070.03005.

[33] V. Lyubashenko and A. Sudbery, Generalized Lie algebras of type $A_{n}$, J. Math. Phys., 39(6) (1998), 3487-3504, MR 99f:17019.

[34] W. Magnus, On the exponential solution of differential equations for a linear operator, Commun. Pure Appl. Math., 7 (1954), 649-673, MR 16,790a, Zbl 0056.34102.

[35] J.W. Milnor and J.C. Moore, On the structure of Hopf algebras, Ann. Math., 81 (1965), 211-264, MR 30 \#4259, Zbl 0163.28202.

[36] S. Montgomery, Hopf Algebras and Their Actions on Rings, CBMS, 82, AMS, Providence, 1993, MR 94i:16019, Zbl 0793.16029.

[37] D.E. Radford, The structure of Hopf algebras with projection, J. Algebra, 92 (1985), 322-347, MR 86k:16004, Zbl 0549.16003. 
[38] M. Rosso, Quantum groups and quantum shuffles, Invent. Math., 113(2) (1998), 399416, MR 2000a:17021, Zbl 0912.17005.

[39] A.I. Shirshov, Subalgebras of free Lie algebras, Matem. Sbornic 33, 75(2) (1953), 441-452 (in Russian).

[40] _ _ On free Lie rings, Matem. Sbornic 45, 87(2) (1958), 113-122.

[41] _ Some algorithmic problems for Lie algebras, Sibirskii Math. J., 3(2) (1962), 292-296.

[42] H. Yamane, A Poincarè-Birkhoff-Witt theorem for quantized universal enveloping algebras of type $A_{N}$, Publ. RIMS. Kyoto Univ., 25 (1989), 503-520, MR 91a:17016, Zbl 0694.17007.

Received February 4, 2000 and revised February 20, 2001. The author was supported by SNI, México, exp. 18740, CONACyT México, Grant 32130-E, PAPIIT UNAM, Grant IN 102599, and in part by the NSF USA Grant DMS-9701755.

Universidad Nacional Autonoma de México

CUAUTITLÁN IZCALLi

Estado DE MÉxico, 54768

MÉXICO

Institute of Mathematics

NovosiBIRSK 630090

Russia

E-mail address: vlad@servidor.unam.mx 